\title{
The Neuroprotective Effects of Carvacrol on Ethanol-Induced Hippocampal Neurons Impairment via the Antioxidative and Antiapoptotic Pathways
}

\author{
Peng Wang, ${ }^{1,2}$ Qian Luo, ${ }^{2}$ Hui Qiao,, ${ }^{2,3}$ Hui Ding, ${ }^{4}$ Yonggang Cao, ${ }^{5}$ \\ Juan Yu, ${ }^{2}$ Ruxia Liu, ${ }^{2}$ Qianlong Zhang, ${ }^{2}$ Hui Zhu, ${ }^{1}$ and Lihui Qu ${ }^{2}$ \\ ${ }^{1}$ Department of Physiology, College of Basic Medical Sciences, Harbin Medical University, Harbin 150081, China \\ ${ }^{2}$ Department of Physiology, College of Basic Medical Sciences, Harbin Medical University-Daqing, Daqing 163319, China \\ ${ }^{3}$ Department of Gastroenterology, Daqing Oilfield General Hospital, Daqing 163319, China \\ ${ }^{4}$ Department of Anesthesiology, Daqing Fifth Hospital, Daqing 163319, China \\ ${ }^{5}$ Department of Pharmacology, College of Basic Medical Sciences, Harbin Medical University-Daqing, Daqing 163319, China
}

Correspondence should be addressed to Hui Zhu; dzhuhui@aliyun.com and Lihui Qu; lihui_qu@aliyun.com

Received 7 April 2016; Revised 2 November 2016; Accepted 23 November 2016; Published 16 January 2017

Academic Editor: Shao-Yu Chen

Copyright (C) 2017 Peng Wang et al. This is an open access article distributed under the Creative Commons Attribution License, which permits unrestricted use, distribution, and reproduction in any medium, provided the original work is properly cited.

\begin{abstract}
Chronic alcohol consumption causes hippocampal neuronal impairment, which is associated with oxidative stress and apoptosis. Carvacrol is a major monoterpenic phenol found in essential oils from the family Labiatae and has antioxidative stress and antiapoptosis actions. However, the protective effects of carvacrol in ethanol-induced hippocampal neuronal impairment have not been fully understood. We explored the neuroprotective effects of carvacrol in vivo and in vitro. Male C57BL/6 mice were exposed to $35 \%$ ethanol for 4 weeks to establish ethanol model in vivo, and hippocampal neuron injury was simulated by $200 \mathrm{mM}$ ethanol in vitro. Morris water maze test was performed to evaluate the cognitive dysfunction. The oxidative stress injury of hippocampal neurons was evaluated by measuring the levels of oxidative stress biomarkers. Histopathological examinations and western blot were performed to evaluate the apoptosis of neurons. The results showed that carvacrol attenuates the cognitive dysfunction, oxidative stress, and apoptosis of the mice treated with ethanol and decreases hippocampal neurons apoptosis induced by ethanol in vitro. In addition, western blot analysis revealed that carvacrol modulates the protein expression of Bcl-2, Bax, caspase-3, and pERK, without influence of $\mathrm{p}$-JNK and p-p38. Our results suggest that carvacrol alleviates ethanol-mediated hippocampal neuronal impairment by antioxidative and antiapoptotic effects.
\end{abstract}

\section{Introduction}

It is well known that ethanol is a deleterious agent which can damage many organs and cause serious health problems [1-4]. Long-term excessive consumption of ethanol leads to behavioral changes, addiction, hyperactivity, mental retardation, depression, and cognitive dysfunction [5-7]. Studies demonstrated that ethanol exposure reduces hippocampal volume, decreases glucose metabolism of cerebrum, and cerebral blood flow and has effects on several neurotransmitter systems that may contribute to cognitive deficits [812]. However, less is known about the detailed mechanism of the effects of ethanol on hippocampal neurons damage. Oxidative stress has been considered as the most plausible cause of ethanol-induced neuronal damage [13-15]. Ethanol promotes production of lipid peroxidation, increases reactive oxygen species (ROS), decreases the activity of antioxidant enzymes, and augments oxidative stress [16-18]. Furthermore, the imbalance of oxidation and antioxidation activates apoptotic cascades by mitochondrial signaling pathway [1921]. In addition, cumulative evidences indicated that ethanolinduced oxidative stress also participates in the modulation of the mitogen-activated protein kinase (MAPK) pathways $[22,23]$. 

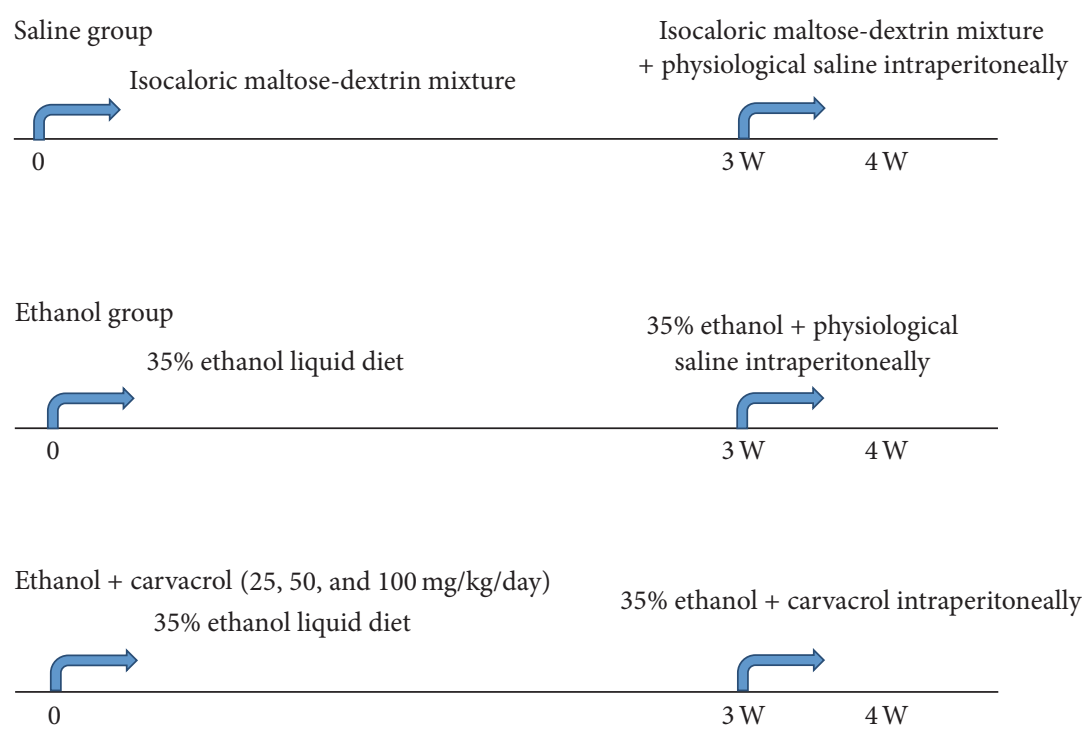

FIGURE 1: Scheme of the in vivo experimental design.

Carvacrol $\left[\mathrm{CAR}, \mathrm{C}_{6} \mathrm{H}_{3}(\mathrm{OH})\left(\mathrm{C}_{3} \mathrm{H}_{7}\right)\right.$ ] is a natural component found in various plants of the family Lamiaceae, including the genera Origanum and Thymus. [24]. It has been widely used as an ingredient of human diet in food or food additive in the food industry for long time. In recent years, several studies have reported that carvacrol has multiple physiological actions, including antibacterial and antifungal $[25,26]$, anti-inflammatory [27], and antitumor activities [28].

Aside from these above pharmacological effects, potential protective effect of carvacrol also has been observed to the central nervous system diseases through diverse mechanisms, including anxiolytic effect, and inhibitory effect on acetylcholinesterase activity [29]. In addition, a recent study demonstrated that carvacrol presented its neuroprotective effect against cerebral ischemia/reperfusion injury in mice [30]. However, there are no detailed reports regarding a potential protective effect of carvacrol against ethanolmediated hippocampal neuronal impairment in C57BL/6 mice. Therefore, the present study was conducted to evaluate the neuroprotective potential of carvacrol against ethanol-mediated hippocampal neuronal impairment, as well as further explore whether neuroprotection of carvacrol was associated with antioxidation and antiapoptosis with the mitochondrial and MAPK signaling pathway involved.

\section{Methods}

2.1. Animals and Treatments. Male C57BL/6 mice $(25 \pm$ $0.5 \mathrm{~g})$ were allowed to acclimatize $(12: 12 \mathrm{~h}$ light/dark cycle, $24^{\circ} \mathrm{C}$ ) for 7 days. The use of the animal tissue was approved by the Ethics Committee of Laboratory Animals at Harbin Medical University. A summary of the experimental design is provided in Figure 1. Mice were randomly assigned to 2 groups including the control diet (saline group) and ethanol diet group. The groups were pair-fed liquid diets containing (in percent of energy intake) 18\% protein, 35\% fat, $12 \%$ carbohydrate, and 35\% either ethanol (ethanol diet) in ethanol group or an isocaloric maltose-dextrin mixture (control diet) in saline group for 4 weeks [31]. Then, after 3 weeks of control diet treatment, saline group mice were treated with control diet and vehicle intraperitoneally (physiological saline $0.1 \mathrm{~mL} / 100 \mathrm{~g} /$ day) for 7 days. The ethanol diet mice were divided into 4 groups as follows. (1) Ethanol group: mice were treated with ethanol diet for 3 weeks; the following 7 days mice were treated with ethanol diet and vehicle intraperitoneally (physiological saline $0.1 \mathrm{~mL} / 100 \mathrm{~g} /$ day); (2) Ethanol + Carvacrol groups: mice were treated with ethanol diet for 3 weeks, and then the mice were subjected to ethanol diet and carvacrol intraperitoneally at 25, 50, and $100 \mathrm{mg} / \mathrm{kg} /$ day, respectively, for 7 days. After the endpoint of treatments, mice were used for the Morris water maze, morphological experiments, oxidative stress measurements, and western blot. Carvacrol (cat\# W224502) was purchased from Sigma-Aldrich, USA.

2.2. Blood Ethanol Concentration. To determine blood ethanol concentration (BEC), blood was collected from tail once a week. Tail blood samples were centrifuged to obtain plasma, and the ethanol concentration was measured with an Analox AM1 analyzer (Analox Instruments; Lunenburg, MA, USA, cat\# GMRD-023).

2.3. Morris Water Maze. After 4 weeks of ethanol consumption, 50 mice $(n=10)$ were used for Morris water maze (MWM) test. The experiment was performed in a white circular water tank $(150 \mathrm{~cm}$ diameter and $60 \mathrm{~cm}$ height $)$ with a smooth inner surface. It rendered opaque water at $22 \pm 1^{\circ} \mathrm{C}$ with white synthetic food colors. A $10 \mathrm{~cm}$ square platform was 
located $2 \mathrm{~cm}$ below the water surface. The pool was divided into four quadrants, and the platform was placed at the midpoint of a quadrant. On the 1st day, all mice were allowed to swim freely. On the 2nd-4th day, the mice were pretrained to find the hidden platform. On the 5th-8th day, each mouse was subjected to 4 trails per day in a maximum of $60 \mathrm{~s}$. The time to climb onto the platform was recorded for each trial as escape latency (s). On the 9th day, the platform was removed and the passing times of the mice that crossed the place where the platform was previously located were recorded.

2.4. Nissl Staining. After animals received ethanol or control diet 4 weeks, respectively, the mice in each group $(n=6)$ were anesthetized with $10 \%(\mathrm{v} / \mathrm{v})$ chloral hydrate and transcardially perfused with $0.1 \mathrm{M}$ phosphate buffered saline (PBS, pH 7.4) for $10 \mathrm{~min}$, followed by fixation by $4 \%$ paraformaldehyde in $0.1 \mathrm{M}$ phosphate buffered saline (PB, pH 7.4) for $10 \mathrm{~min}$. The brains were then removed, postfixed in $4 \%$ paraformaldehyde for $48 \mathrm{~h}$, and then cryoprotected by infiltration with $30 \%$ sucrose for 3 days at $4^{\circ} \mathrm{C}$. Coronal sections $(8 \mu \mathrm{m})$ of the hippocampus were cut and submerged in $0.1 \%$ cresyl violet for $10 \mathrm{~min}$ at $37^{\circ} \mathrm{C}$ and then were rinsed in distilled water, dehydrated in graded ethanol, and cover lipped with neutral balsam. For Nissl staining, the normal neurons were round, and the nuclei appeared as pallid blue. In the ethanoltreated group, the cells were shrunken, with nuclei pyknosis. Quantitative assessment of the number of live neurons was evaluated from $10 \mathrm{CA} 1$ region per brain sample $(n=6)$ by Image-Pro Plus 6.0 (Media Cybernetics, Bethesda, MA, USA).

2.5. NeuN Immunohistochemistry. Hippocampus injury was evaluated based on the results of Nissl staining and immunohistochemistry in brain sections. Tissue sections were treated with $0.3 \%$ hydrogen peroxide $\left(\mathrm{H}_{2} \mathrm{O}_{2}\right)$ for $10 \mathrm{~min}$ and then nonspecific antibody binding was blocked with $10 \%$ goat serum for $30 \mathrm{~min}$ at room temperature. The sections were incubated with anti-NeuN $(1: 200$, Chemicon, CA) overnight at $4^{\circ} \mathrm{C}$, and, subsequently, the sections were exposed to biotinylated goat anti-mouse IgG and streptavidin peroxidase complex (Vector, Burlingame, CA) for $30 \mathrm{~min}$ at $37^{\circ} \mathrm{C}$. They were soaked in 3,3-diaminobenzidine (DAB), and the reaction was stopped with distilled water. The stained sections were observed under a light microscope. Quantification of the number of NeuN-immunopositive cells from 10 CA1 region per brain sample $(n=6)$ was by Image-Pro Plus 6.0 (Media Cybernetics, Bethesda, MA, USA).

2.6. Double Immunofluorescence Staining. Immunofluorescent double staining of NeuN and TUNEL was performed to explore colocalization of apoptotic cells and neurons. The hippocampal tissue sections were treated the same way as experimental procedure in Section 2.4 before TUNEL and NeuN staining. TUNEL staining was conducted according to the manufacturer's protocol of In Situ Cell Death Detection Kit (Roche, Germany), followed by antibody staining against NeuN (1:200, Chemicon, CA). Finally, the sections were observed under a fluorescence microscope (Leica, Germany).

2.7. Hippocampal Neurons Culture. Neonatal rat hippocampal neurons were prepared from 2- to 3-day-old neonatal C57BL/6 mice. The hippocampi were dissected and chopped into $1 \mathrm{~mm}^{3}$ pieces under a light microscope. The hippocampal chunks were digested by $0.125 \%(\mathrm{w} / \mathrm{v})$ trypsinase at $37^{\circ} \mathrm{C}$ for 10 min and then resuspended in DMEM supplemented 10\% fetal bovine serum (FBS) in order to stop trypsin activity. After centrifugation at $14,000 \mathrm{~g}$ for $5 \mathrm{~min}$, the supernatant was discarded and the cell pellet was resuspended in DMEM. Hippocampal cultures were maintained in serum-free Neurobasal medium, containing B27 supplement in a humidified atmosphere ( $95 \%$ air, $5 \% \mathrm{CO}_{2}$ ) at $37^{\circ} \mathrm{C}$. Hippocampal neurons were chosen for experiments between days 8 and 14 of culture. Hippocampal neurons were randomly divided into 4 groups: (1) control group; (2) model group, cells were incubated with $200 \mathrm{mM}$ ethanol for $24 \mathrm{~h}$; (3) model + carvacrol group, cells were given $0.8 \mathrm{mM}$ carvacrol for $4 \mathrm{~h}$ after treatment with $200 \mathrm{mM}$ ethanol for $24 \mathrm{~h}$; (4) control + carvacrol group, cells were given $0.8 \mathrm{mM}$ carvacrol for $4 \mathrm{~h}$.

2.8. Hippocampal Neurons Viability Assay. Hippocampal neurons were plated at 30,000 cells/well in a 96-well plate. Cell viability was assessed at day 2 of cell culture. According to the manufacturer's recommendations, $40 \mu \mathrm{L}$ MTS solution was added to each of the wells, and absorbance was obtained at $490 \mathrm{~nm}$ using a microplate reader (FlexStation 3; Molecular Devices, Sunnyvale, CA, USA). The same volume of medium without cells was used as blank.

2.9. Hoechst Staining. Hippocampal neurons were grown in six-well plates. After drug treatments, cells were fixed with $4 \%$ paraformaldehyde for $4^{\circ} \mathrm{C}$ overnight. Fixed cells were washed with PBS three times and stained with Hoechst 33258 (final concentration, $0.5 \mathrm{ug} / \mathrm{mL}$ ) for $5 \mathrm{~min}$. The six-well plates were visualized using a fluorescent microscope (IX51, Olympus).

2.10. Oxidative Stress Measurements. Mice were sacrificed after treatment of ethanol or control diet for 4 weeks, respectively. The hippocampus was rapidly excised from the brain and kept frozen at $-80^{\circ} \mathrm{C}$. Half of the tissue was taken for oxidative stress measurements and the other half of the section was detected for western blot analysis. The enzymatic activities of CuZn-superoxide dismutase (SOD), Mn-SOD, SOD, glutathione (GSH), glutathione peroxidase (GSH-PX), and catalase (CAT) and the level of malondialdehyde (MDA) were evaluated with different detection kits according to the manufacturer's instructions (Nanjing Jian Cheng Bioengineering Institute). One part of the tissues which was used for oxidative stress measurements was weighed and rinsed with cold isotonic saline. Hippocampus homogenate $(10 \%$, $\mathrm{w} / \mathrm{v}$ ) was prepared by homogenizing the hippocampus tissue in cold saline ( $\mathrm{pH}$ 7.0). CuZn-SOD and Mn-SOD activities were detected in hippocampus homogenate by measuring 
the ability to inhibit the photochemical reduction of nitro blue tetrazolium (NBT) in absorbance at $550 \mathrm{~nm}$. Data were recorded as SOD units/mg protein. The level of MDA in the hippocampus was calculated by measuring thiobarbituric acid reacting substances at $532 \mathrm{~nm}$. The concentration of malondialdehyde was expressed as $\mathrm{nmol} / \mathrm{mg}$ protein. The activities of GSH-PX and GSH were detected by quantifying the rate of reduced glutathione to the oxidized glutathione. The levels of enzymatic activities were measured with a spectrophotometer (U-2000, Hitachi).

2.11. Western Blot. Western blot analysis was performed on the hippocampal tissues. Briefly, tissues were lysed with RIPA buffer (Beyotime Institute of Biotechnology, China) containing $50 \mathrm{mM}$ Tris, $150 \mathrm{mM} \mathrm{NaCl}, 1 \%$ Triton X-100, $1 \mathrm{mM}$ EDTA, and $2 \mathrm{mM}$ PMSF containing protease inhibitors for $30 \mathrm{~min}$ on ice. The lysates were centrifuged at $13,500 \mathrm{~g}$ for $15 \mathrm{~min}$ at $4^{\circ} \mathrm{C}$, and the supernatant was collected and total protein content was determined using a BCA protein assay kit with bovine serum albumin as the standard (Beyotime Institute of Biotechnology, China). Samples with an equal amount of protein $(50 \mu \mathrm{g})$ were separated in $10 \%$ or $12 \%$ SDSpolyacrylamide gels and then transferred onto nitrocellulose membranes (Millipore, MA). The membranes were then blocked using 5\% fat-free milk in Tris-buffered saline with $1 \%$ Tween-20 (TBS-T) for $1 \mathrm{~h}$ and then were incubated with the following incubation with primary antibodies overnight at $4^{\circ} \mathrm{C}$ : anti-caspase-3 (1:300, Santa Cruz), anti-Bcl-2 (1:200, Santa Cruz), anti-Bax $(1: 200$, Santa Cruz), anti-phosphoERK (1:1000, Cell Signaling), anti-ERK (1:1000, Cell Signaling), anti-phospho-JNK (1:200, Santa Cruz), anti-JNK (1:200, Santa Cruz), anti-phospho-p38 (1:200, Santa Cruz), anti-p38 ( $1: 200$, Santa Cruz), and anti- $\beta$-actin ( $1: 2000$, Santa Cruz), respectively. The membranes were washed with TBS$\mathrm{T}$, followed by the incubation with horseradish peroxidaseconjugated goat anti-rabbit-antibody (1:5000, Santa Cruz) or goat anti-mouse antibody (1:5000, Santa Cruz), for $2 \mathrm{~h}$ at room temperature. Immunoreactive bands were visualized by enhanced chemiluminescence (ECL) kit (Pierce, CA) and exposed on an X-ray film. The immunoblots intensities were quantified using the Quantity One software (BioRad).

2.12. siRNA Transfection. To silence the expression of ERK1/2 protein, hippocampal neurons were transfected with small interfering RNA, which was designed and synthesized by GenePharma (Shanghai, China). Nontargeted control siRNA (si-NC) was used as negative control. The sense sequence of siRNA against ERK1/2 and nontargeted control sequence were listed below: accession No.ds-siRNA sequence corresponding nucleotides, ERK1: $5^{\prime}-\mathrm{G}$ G A C C A G C U C A A C C AC A U U-3', ERK2: 5'G C U C U U G A A G A C A C A G C A C$3^{\prime}$ NC control: 5'-UUCUCCGAACGUGUCACGUTT-3'. Briefly, the hippocampal neurons were cultured till 30\%$50 \%$ confluence and then $2 \mathrm{ug}$ siRNA and $10 \mathrm{ul} \mathrm{X-tremeGene}$ siRNA Transfection Reagent were, respectively, diluted in serum-free Opti-MEM-1 medium and mixed. The mixture
(siRNA/Transfection Reagent) was incubated at room temperature for $20 \mathrm{~min}$ and added directly onto cells. After transfections, cells were quiesced for $48 \mathrm{~h}$ and used as required.

2.13. Flow Cytometric Analysis. The apoptosis rate of hippocampal neurons was carried out by flow cytometric analysis. Both attached and floating hippocampal neurons were harvested and washed twice with PBS, fixed in ice-cold $80 \%$ ethanol, and stored overnight at $4^{\circ} \mathrm{C}$. Then, the cells were washed twice with PBS and $10 \mathrm{mg} / \mathrm{mL}$ RNase A was added. FITC-labeled Annexin V/PI staining was performed according to the manufacturer's instructions (Keygen, Nanjing, China). For each experiment, 20,000 cells were analyzed using Elite Flow Cytometry (BD Biosciences, San Jose, CA) and Cell Quest software (BD Biosciences). Triplicates were performed in all cases for the detection of early apoptotic cells.

2.14. Caspase-3 Activity Assay. A colorimetric assay kit (Beyotime, Jiangsu, China) was used to evaluate the activity of caspase-3. Briefly, hippocampal neurons were treated the same way as experimental procedure in Section 2.11 and then were centrifuged at $4^{\circ} \mathrm{C}$ for $20 \mathrm{~min}$, the supernatant was collected. Approximately $1-3 \mu \mathrm{g} / \mathrm{mL}$ protein was incubated with substrate Ac-DEVD-pNA (acetyl-Asp-Glu-Val-Aspnilide) at $37^{\circ} \mathrm{C}$ for $2 \mathrm{~h}$. The absorbance of pNA was measured with a spectrophotometer at $405 \mathrm{~nm}$. The activity of caspase- 3 was normalized for total protein and expressed as fold of the baseline caspase- 3 activity of the control group.

2.15. Statistical Analysis. Data were represented as mean \pm SEM. Statistical analysis was carried out using one-way ANOVA followed by Turkey test for individual comparisons between group means. All statistical analyses were performed by SPSS 19.0 software. $P<0.05$ was considered statistically significant.

\section{Results}

3.1. Effect of Carvacrol on Body Weight and Blood Ethanol Levels. 4 weeks after ethanol diet, mice exhibited significantly decreased body weights compared with the saline group $(P<$ 0.05). However, chronic treatments with carvacrol at the doses of 25,50 , and $100 \mathrm{mg} / \mathrm{kg}$ displayed no influence with the blood ethanol levels and body weights of mice, compared with the ethanol group $(P>0.05)$ (Figures 2(a) and 2(b)). The results indicated that carvacrol does not affect blood ethanol concentration and body weight of mice.

3.2. Carvacrol Improved Ethanol-Induced Cognitive Deficits. Cognitive function was assessed with the Morris water maze test. By one-way ANOVA analysis, as shown in Figure 3(a), the escape latency of the ethanol-treated group significantly increased in comparison to that in the saline group $(P<$ $0.01)$, while carvacrol treated animals at dose of 50 and $100 \mathrm{mg} / \mathrm{kg}$ reversed this effect $(P<0.01)$. There was no significant difference between ethanol and carvacrol $25 \mathrm{mg} / \mathrm{kg}$ treatment group $(P>0.05)$. Likewise, Figure $3(\mathrm{~b})$ also indi- 


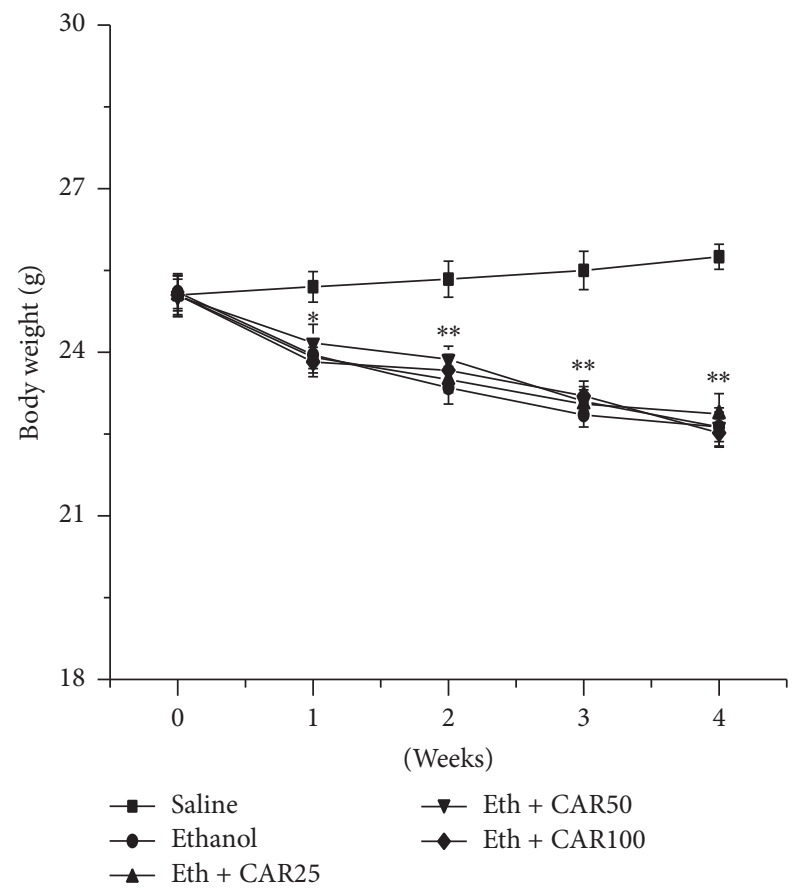

(a)

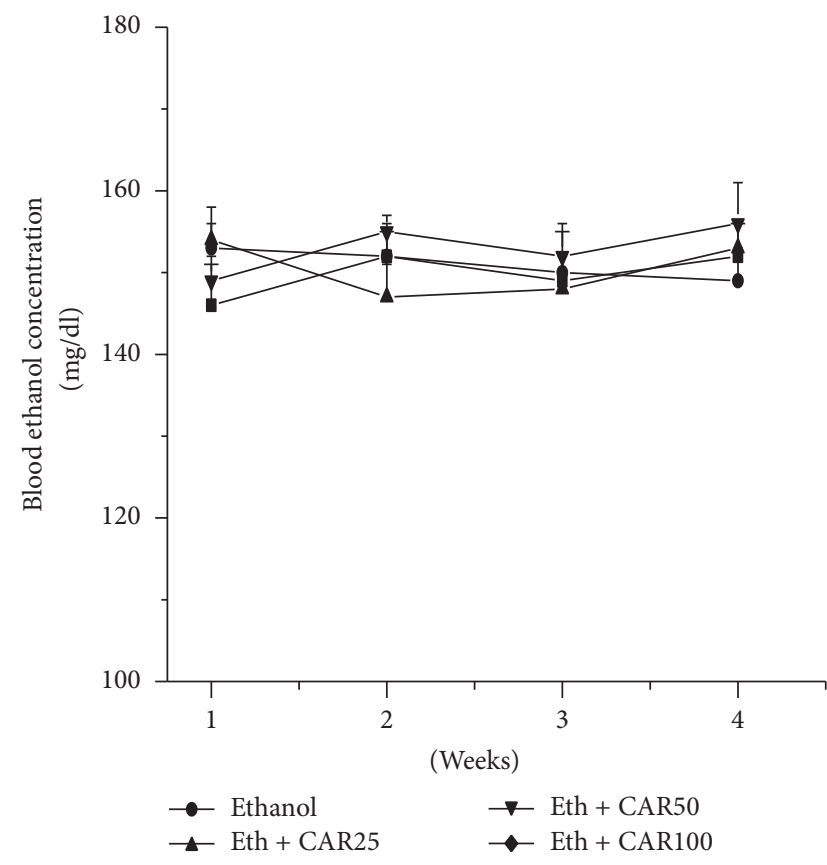

(b)

FIGURE 2: (a) Body weight changes of mice during 4 weeks liquid diets treatment. No significant differences were detected between ethanol and ethanol $+\operatorname{CAR}(25,50$, and $100 \mathrm{mg} / \mathrm{kg} /$ day) groups. (b) Blood ethanol concentration in different groups of mice during 4 weeks treatment. No significant effect of carvacrol and no significant interaction between ethanol and carvacrol in blood ethanol concentration. The data are expressed as the mean $\pm \operatorname{SEM}\left(n=6\right.$ per group). ${ }^{*} P<0.05$ and ${ }^{* *} P<0.01$ compared to saline group.

cated an evident increase in mean path length in ethanoltreated group; this phenomenon was obviously prevented after treatment with carvacrol in a dose-dependent way $(P<0.01)$. The evident decreases for the time stayed in the target quadrant and the number of times the animals crossed the former platform location were observed in ethanoltreated group versus saline group $(P<0.01)$, as shown in Figures 3(c) and 3(d), respectively. However, supplement with carvacrol (50 and $100 \mathrm{mg} / \mathrm{kg}$ ) dose-dependently reversed the two indices $(P<0.01)$.

3.3. Carvacrol Protected Hippocampal Neuron against Ethanol-Induced Apoptosis. In order to explore the effects of carvacrol on neuron apoptosis induced by chronic ethanol feeding in hippocampal tissues, histological examination of Nissl staining was performed to evaluate a significant loss of ethanol-induced hippocampal CA1 region neurons. As illustrated in Figure 4(a), neurons in the saline group did not have any histopathological abnormalities; however, extensively damaged neurons with pyknotic nuclei were observed in ethanol-treated mice. Carvacrol treatment markedly increased cell survival with palely stained nuclei in comparison with the ethanol group $(P<0.01)$. NeuN immunohistochemistry showed that the number of NeuN-immunopositive cells in the hippocampal CA1 region was evidently decreased in the ethanol group compared with the saline group as shown in Figure 4(b). Carvacrol treatment animals at doses of 50 and $100 \mathrm{mg} / \mathrm{kg}$ markedly prevented ethanol-induced neuronal damage $(P<0.01)$. Double-label staining of TUNEL and NeuN showed that the number of NeuN-TUNEL-positive cells was increased in the ethanol group compared with the saline group $(P<0.01)$. The ethanol group mice treated with carvacrol had significantly $(P<0.01)$ less NeuN-TUNEL-positive cells in the hippocampal CA1 region than the mice in ethanol group had (Figure 5).

To detect the antiapoptosis of carvacrol against ethanol toxicity in hippocampal neurons, the best concentrations of carvacrol and ethanol were explored using MTT assay. As shown in Figure 6(a), hippocampal neurons treated with ethanol $(0-300 \mathrm{mM})$ revealed a dose-dependent reduction in the levels of cell viability. $200 \mathrm{mM}$ of ethanol caused about $30 \%$ hippocampal neurons apoptosis. Therefore, the addition of $200 \mathrm{mM}$ ethanol was used in the subsequent experiments. Our results also revealed that carvacrol $(0-0.8 \mathrm{mM})$ did not exhibit a significant toxic effect on the neurons (Figure 6(b)). As shown in Figure 6(c), $0.8 \mathrm{mM}$ of carvacrol significantly increased cell viability compared with ethanol group $(P<$ 0.01 ). Thus, the administration of $0.8 \mathrm{mM}$ of carvacrol was used in the subsequent experiments. This result was confirmed by Hoechst 33342 staining (Figure 6(d)). 

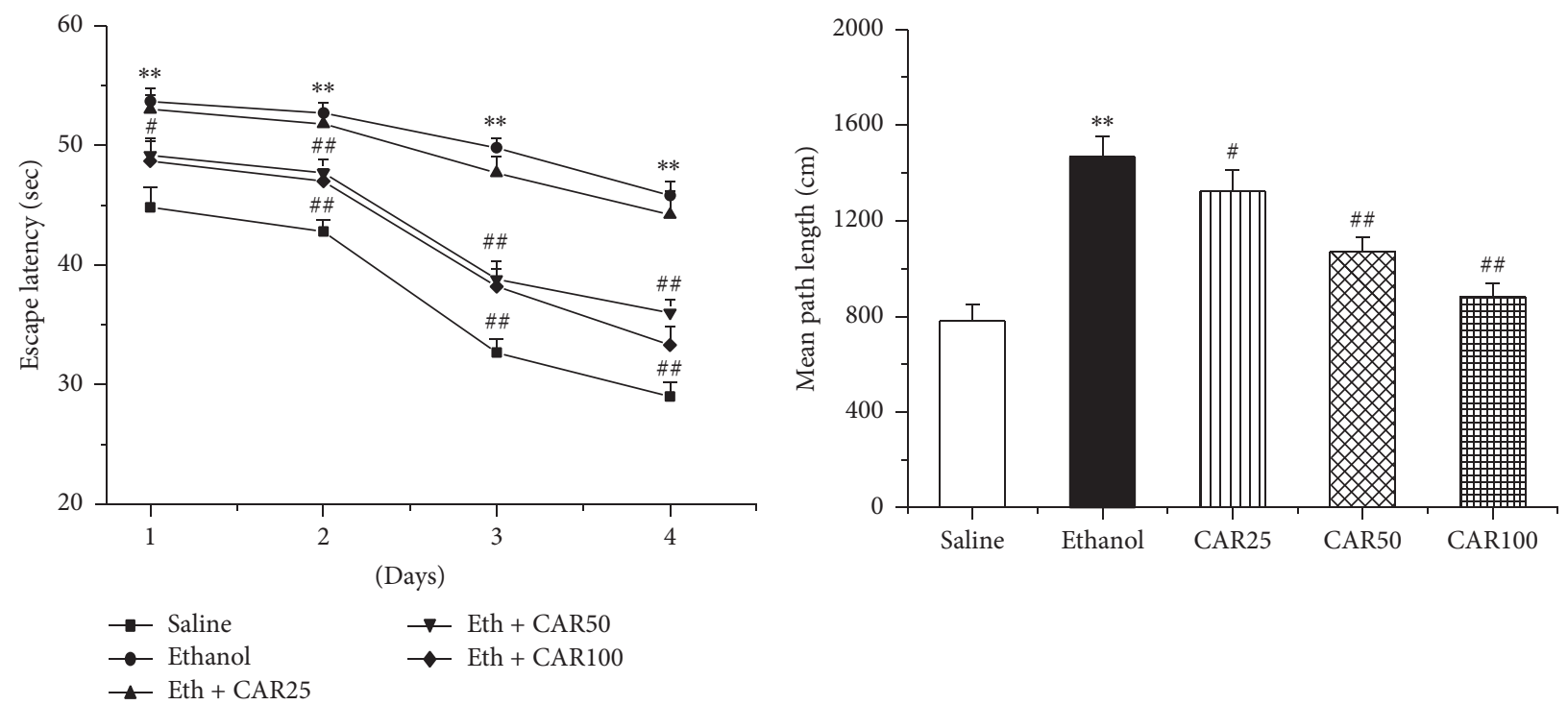

(a)

(b)
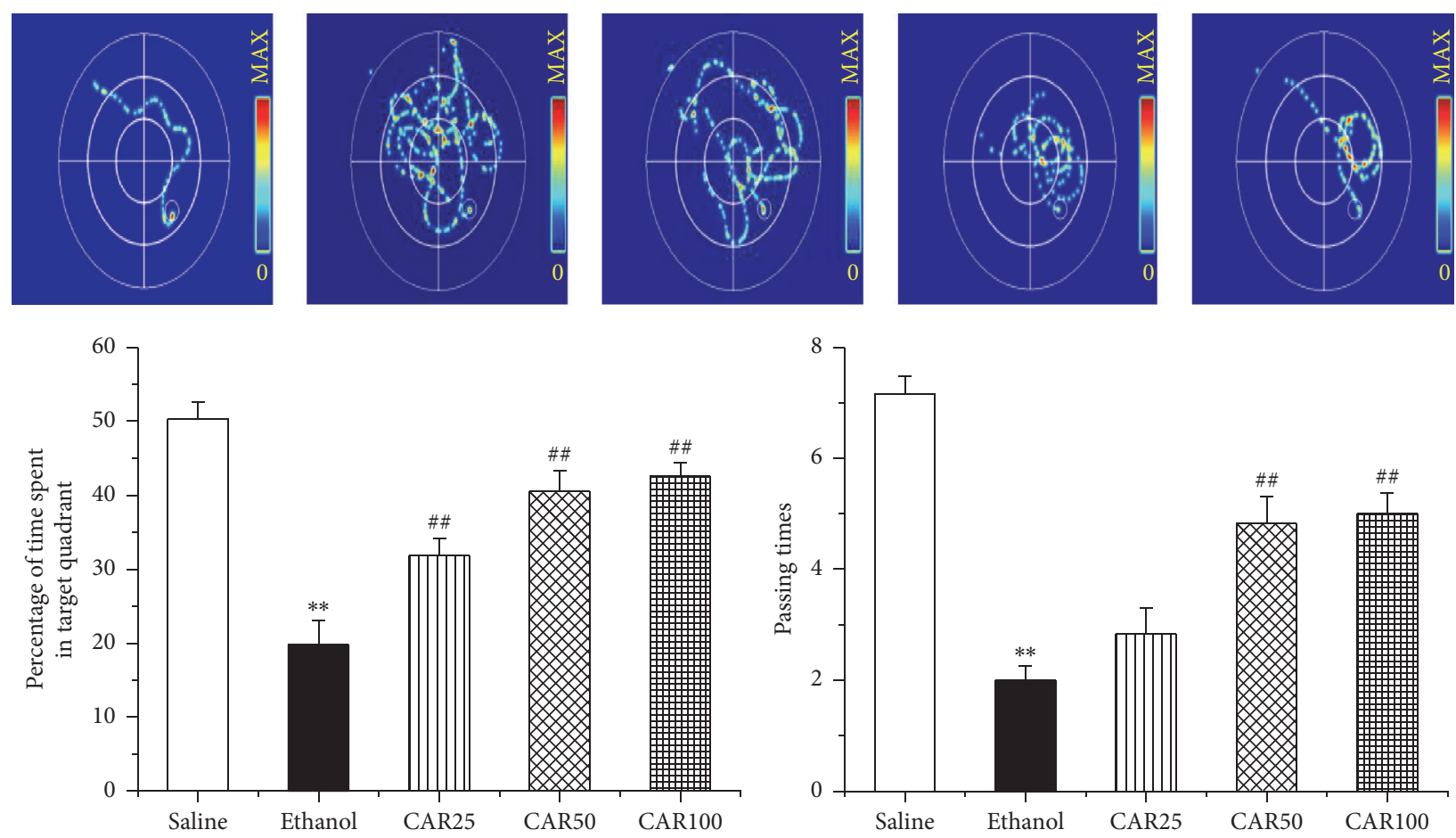

(c)
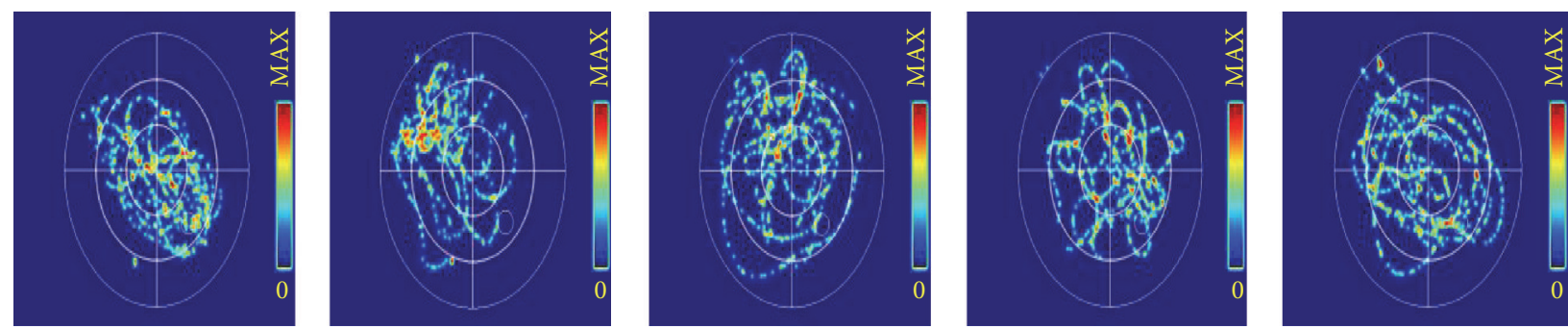

FIGURE 3: Carvacrol ameliorated ethanol-induced cognitive impairment in C57BL/6 mice. Escape latency (a), mean path length (b), mice that stayed in the target quadrant (c), and passing times (d) are shown in the saline, ethanol, and different carvacrol dose groups. CAR25, CAR50, and CAR100 indicate carvacrol 25, 50, and $100 \mathrm{mg} / \mathrm{kg}$-treated groups. The data are expressed as the mean \pm SEM ( $n=10$ per group). ${ }^{* *} P<0.01$ compared to saline group; ${ }^{\#} P<0.05$ and ${ }^{\# \#} P<0.01$ compared to ethanol group. 

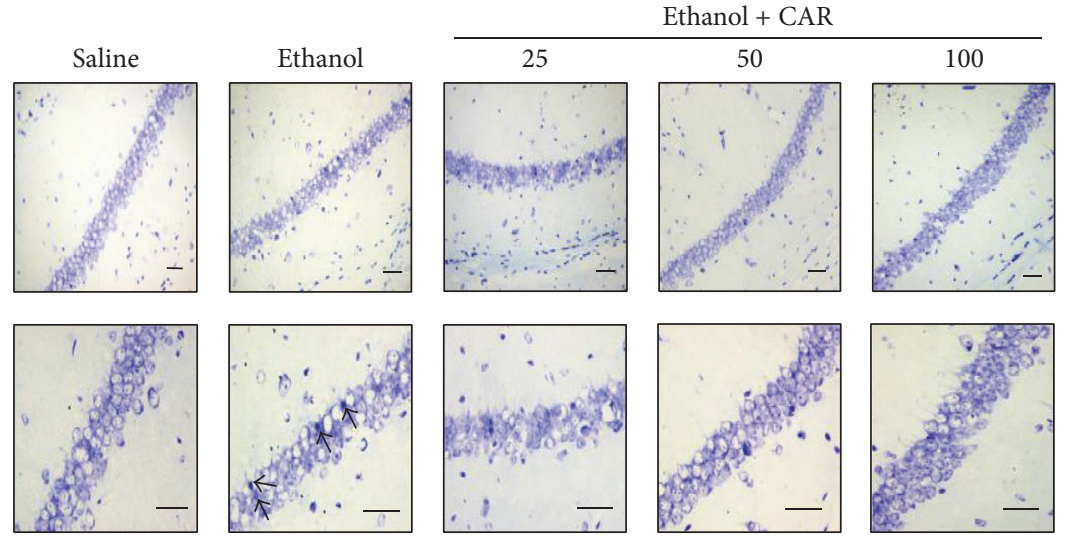

(a)

Ethanol + CAR
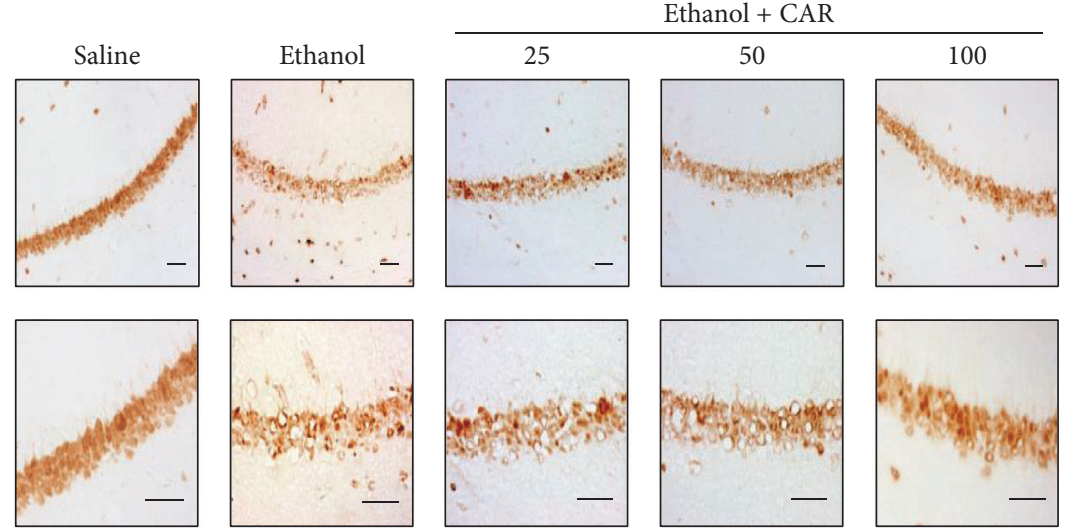

(b)
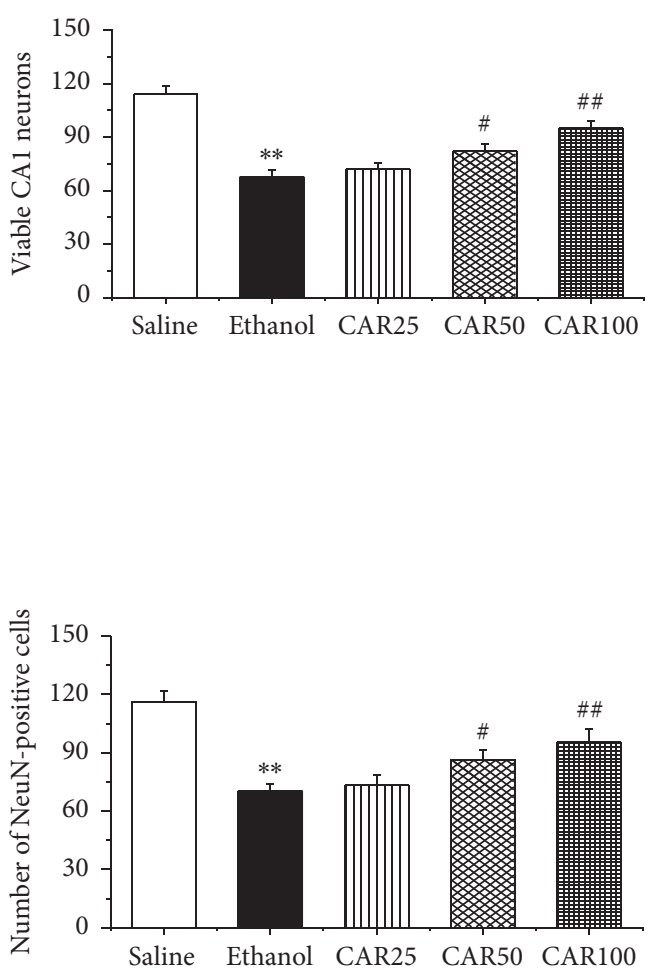

FIGURE 4: Nissl staining and NeuN immunohistochemistry showed the protective effects of carvacrol on ethanol-induced neuronal impairment of the hippocampal CA1 region in C57BL/6 mice. (a) Representative photomicrographs of Nissl staining for surviving neurons in hippocampal CAl region, and the statistical analysis of the surviving cells in each group. Arrows indicated the shrunk dark damaged neurons. (b) Representative immunohistochemical photomicrographs of NeuN in mouse hippocampus, and the statistical analysis of the NeuN-immunopositive cells in each group. There were fewer NeuN and Nissl-positive neurons in the ethanol group than in the saline group. With treatment with different doses of carvacrol, NeuN and Nissl-positive neurons were abundant in the CAl region compared with the ethanol group. Up panel is lower magnification image (200x) and down panel is higher magnification image (400x) of CAl pyramidal neurons. Scale bar: $50 \mu \mathrm{m}$. The data are expressed as the mean $\pm \operatorname{SEM}\left(n=6\right.$ per group). ${ }^{* *} P<0.01$ compared to saline group; ${ }^{\#} P<0.05$ and ${ }^{\# \#} P<0.01$ compared to ethanol group.

3.4. Carvacrol Inhibited Oxidative Stress in Hippocampus of Ethanol-Fed Mouse. To explore whether carvacrol protects the neuronal impairment via antioxidant mechanisms, oxidative stress biomarkers were evaluated in our study. As shown in Figure 7, our results indicated that the ethanol exposure reduced the activities of the antioxidative enzymes and antioxidants compared to the saline group $(P<0.01)$, including CuZn-SOD, Figure 7(a), Mn-SOD, Figure 7(b), SOD, Figure 7(c), GSH, Figure 7(d), GSH-PX, Figure 7(e), and CAT, Figure 7(f). Treatment with carvacrol at both of 50 and $100 \mathrm{mg} / \mathrm{kg}$ significantly reversed the above changes. The concentration of MDA, an index of lipid peroxidation, is depicted. As shown in Figure $7(\mathrm{~g})$, the level of MDA in the ethanol group was significantly increased compared with that of the saline group $(P<0.01)$. Treatment with carvacrol at doses of 50 and $100 \mathrm{mg} / \mathrm{kg}$ suppressed the levels of MDA.

3.5. Carvacrol Increases Bcl-2 Protein Level in Hippocampal Neurons under Ethanol Stimulation. To investigate whether carvacrol modulated the mitochondrial pathway, the expressions of apoptosis-regulatory indices, including caspase-3, $\mathrm{Bcl}-2$, and Bax were detected by western blot. Our results showed that the expressions of caspase- 3 and Bax were remarkably increased but Bcl-2 was decreased in ethanoltreated mice compared with the saline group $(P<0.01)$. 

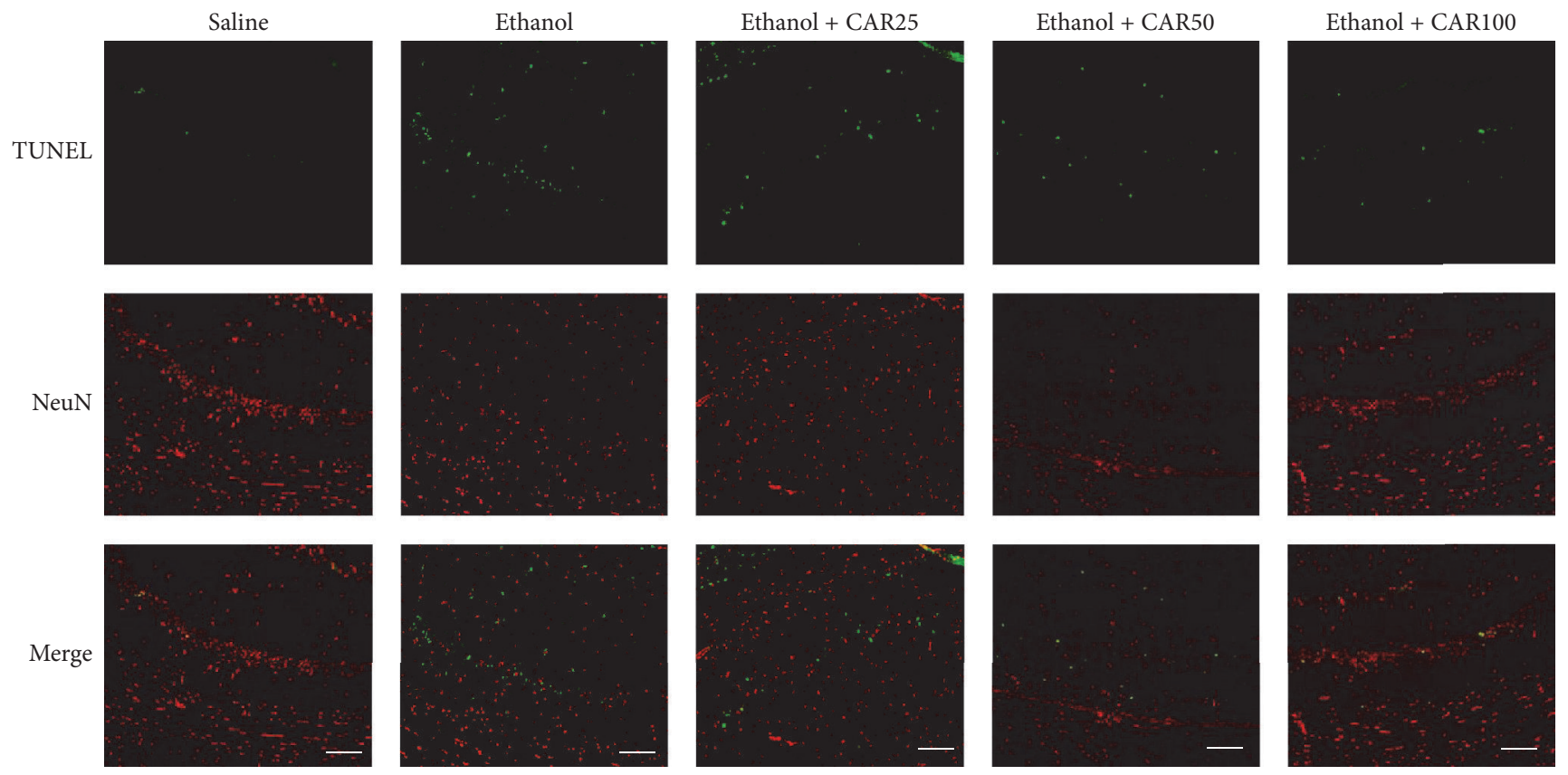

(a)

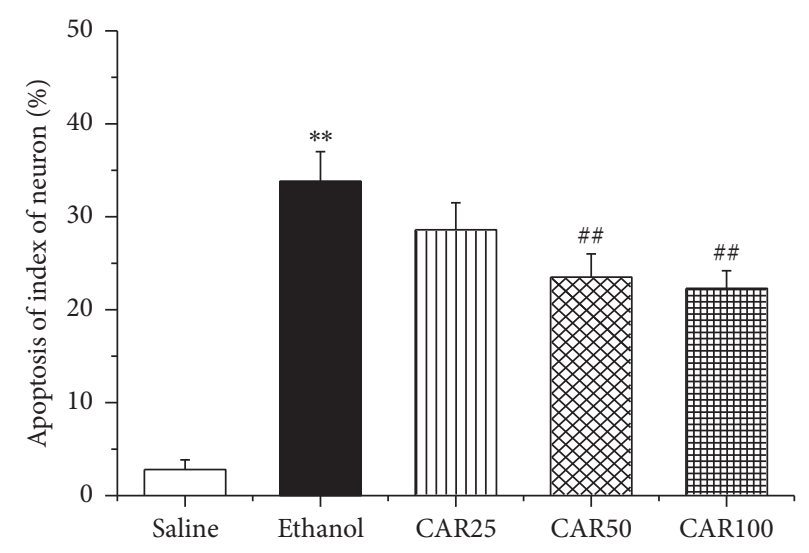

(b)

FIGURE 5: Double fluorescent labeling TUNEL (green) and NeuN (red) showed the protective effects of carvacrol on ethanol-induced neuronal impairment of the hippocampal CA1 region in C57BL/6 mice. (a) Representative photographs of TUNEL and NeuN staining in different groups. TUNEL-positive cells were barely detected in the saline group. In the ethanol group, the apoptotic cells increased markedly compared with the saline group. However, compared with the ethanol group, administration of different doses of carvacrol substantially reduced the number of apoptotic cells. (b) Quantification of TUNEL-positive cells. Scale bar: $100 \mu \mathrm{m}$. The data are expressed as the mean \pm SEM $(n=6$ per group). ${ }^{* *} P<0.01$ compared to saline group; ${ }^{\# \#} P<0.01$ compared to ethanol group.

Administration of 50 and $100 \mathrm{mg} / \mathrm{kg}$ carvacrol decreased Bax and caspase- 3 expression but increased Bcl-2 protein levels compared with the ethanol-treated mice $(P<0.01)$ (Figures $8(\mathrm{a}), 8(\mathrm{~b})$, and $8(\mathrm{c}))$.

3.6. Carvacrol Protects Hippocampal Neurons Apoptosis by MAPKs Signal Pathway. To assess the possible involvement of MAPK activation in the effects of carvacrol on ethanolinduced impairment, we first examined MAPK signal activation in hippocampus by western blotting analysis. As shown in Figure 9(a), ethanol exposure reduced ERK$1 / 2$ protein phosphorylation ( $\mathrm{p}$-ERK-1/2). $\mathrm{p}$-ERK-1/2 was remarkably elevated in the mice treated with carvacrol at doses of 50 and $100 \mathrm{mg} / \mathrm{kg}$ in comparison to the ethanol group. As indicated, the phosphorylation of JNK, Figure 9(b), and p38, Figure 9(c), was markedly increased in ethanoltreated mice and hippocampal neurons, compared to the saline group. However, the carvacrol did not reverse the effect of ethanol on phosphorylation of JNK and p38. 


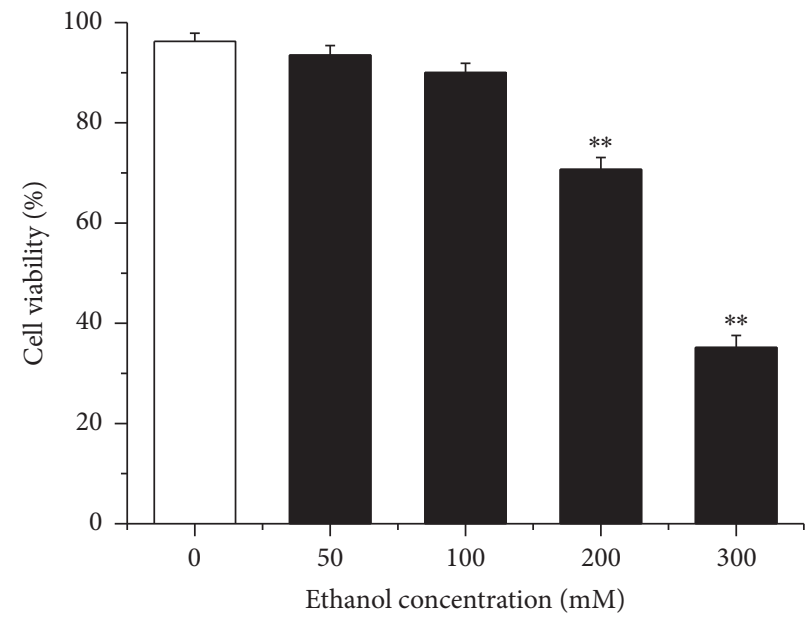

(a)

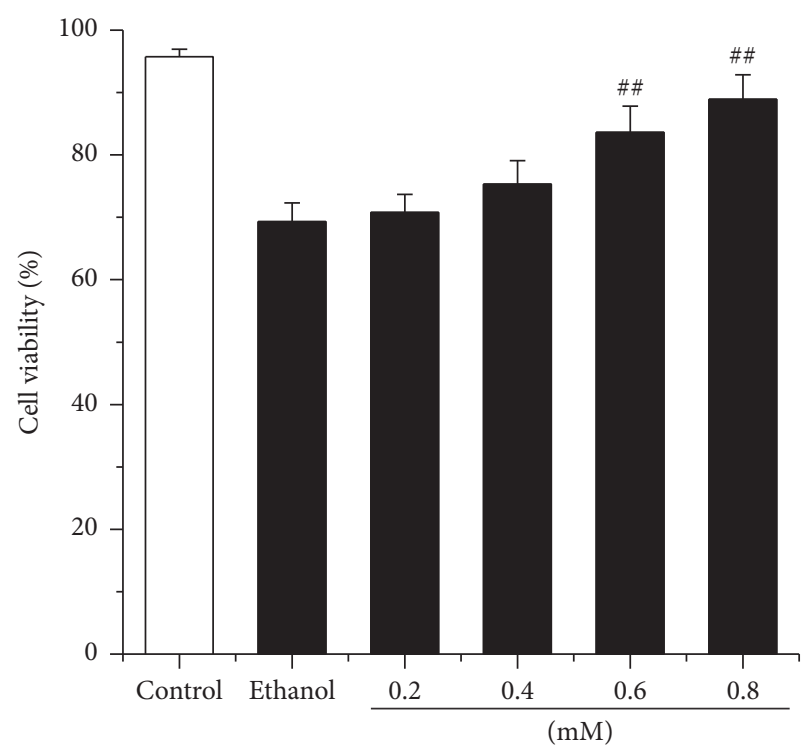

(c)

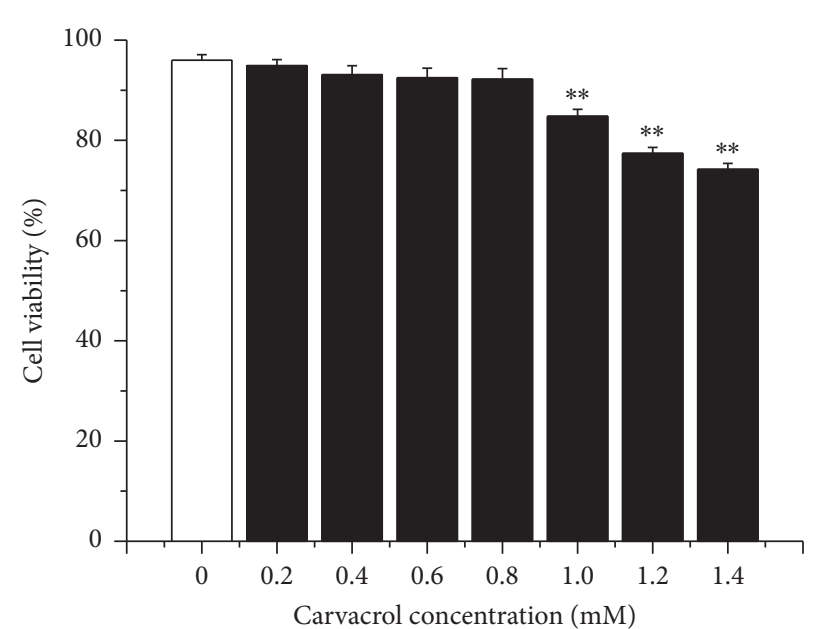

(b)
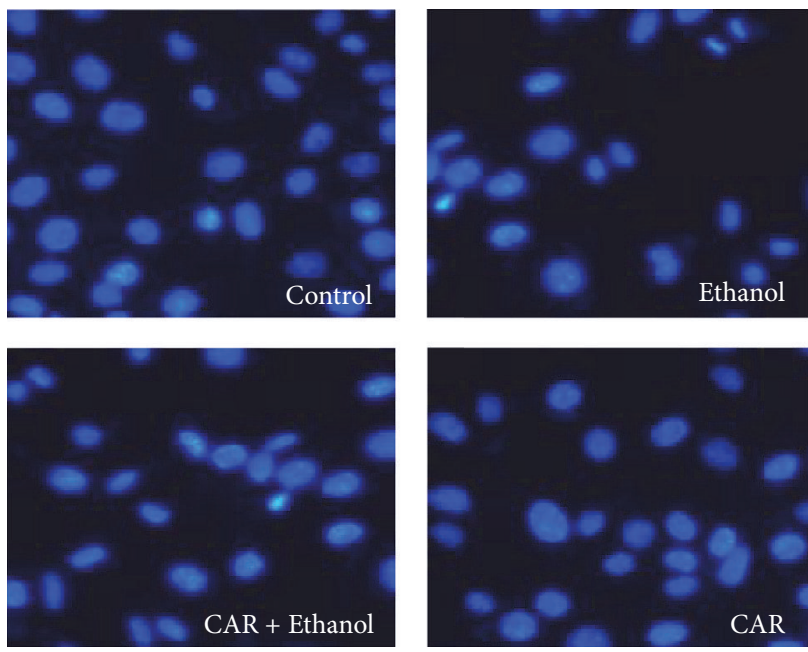

(d)

FIGURE 6: Carvacrol protects cultured hippocampal neurons from ethanol-induced cell death. (a)-(c) To select drug concentrations for the subsequent experiments, MTT assay was carried out on the hippocampal neurons. (a) Primary hippocampal neurons were treated with 0-300 mM ethanol. (b) Hippocampal neurons were treated with 0-1.4 mM carvacrol. (c) Hippocampal neurons were treated with $200 \mathrm{mM}$ ethanol and then exposed to different doses of carvacrol. On the basis of the above results, hippocampal neurons treated with $0.8 \mathrm{mM}$ carvacrol after the incubation of $200 \mathrm{mM}$ ethanol were selected. (d) Hippocampal neurons were incubated with $0.8 \mathrm{mM}$ carvacrol after the incubation of $200 \mathrm{mM}$ ethanol. Cells were observed by fluorescence microscopy after the nuclei were stained with the fluorescent dye Hoechst 33342 . All the values are presented as mean $\pm \operatorname{SEM}\left(n=6\right.$ per group). ${ }^{* *} P<0.01$ compared to control group; ${ }^{\# \#} P<0.01$ compared to ethanol group.

We further silenced ERK1/2 (Figure 10(a)) to confirm carvacrol protective effects through ERK1/2 signaling pathway. Our results revealed that carvacrol significantly decreases hippocampal neurons apoptosis compared to the ethanol group $(P<0.01)$, but these effects can be reversed by silence of ERK1/2 or treatment of U0126 (antagonist of carvacrol) (Figure 10(b)). The caspase-3 activity and cell viability change consistently with the result of hippocampal neurons apoptosis (Figures 10(c) and 10(d)). Taken together, these findings suggested that carvacrol protects hippocampal neurons apoptosis by ERK1/2 signal pathway.

\section{Discussion and Conclusion}

In this study, we demonstrated that carvacrol improves ethanol-mediated cognitive dysfunction of the mice and then 


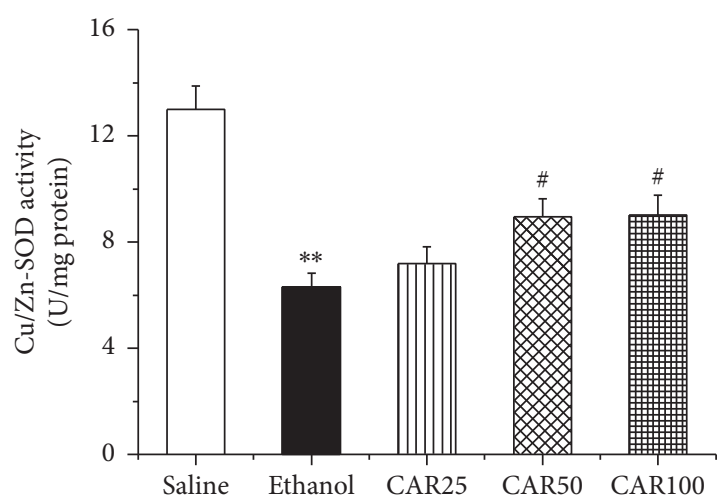

(a)

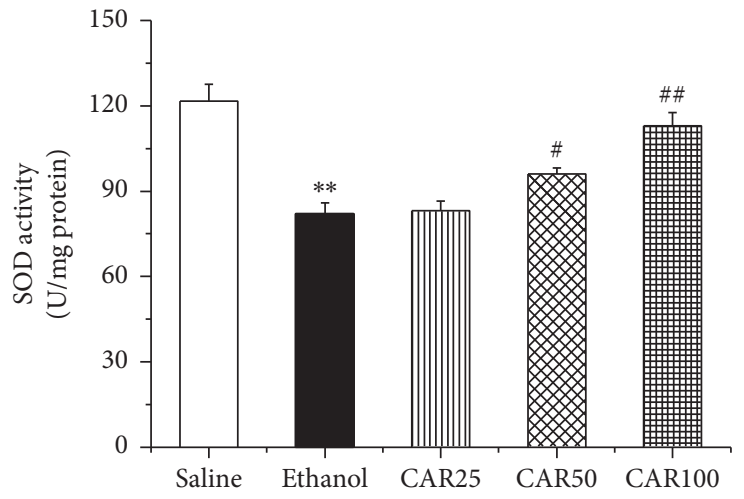

(c)

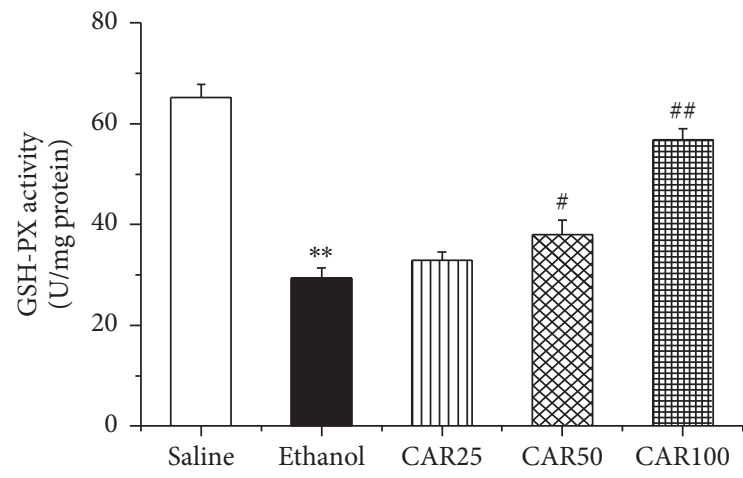

(e)

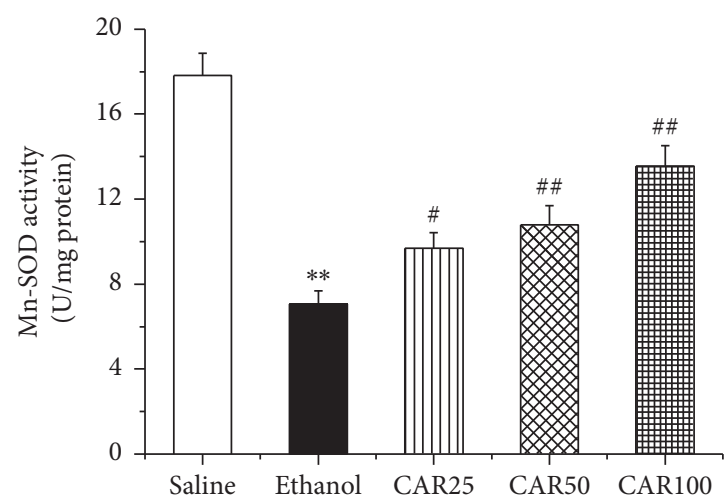

(b)

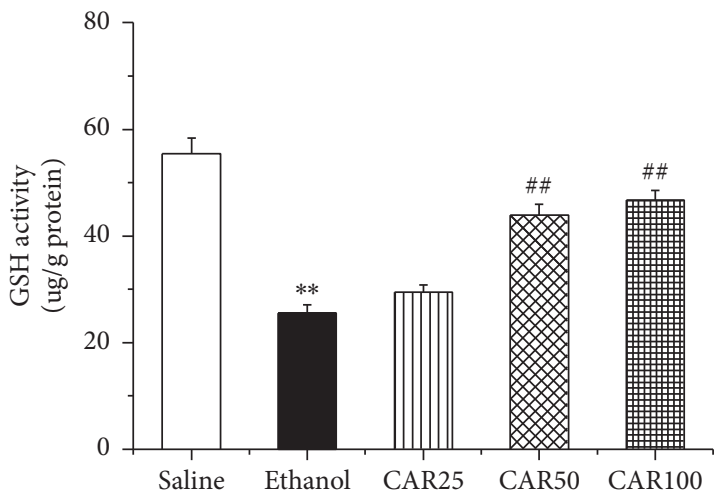

(d)

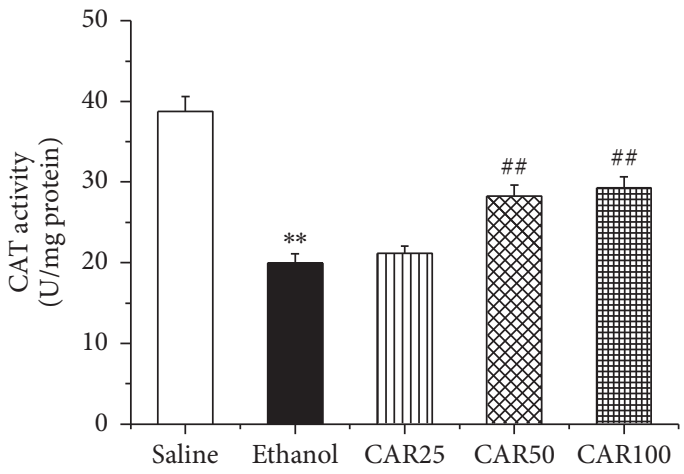

(f)

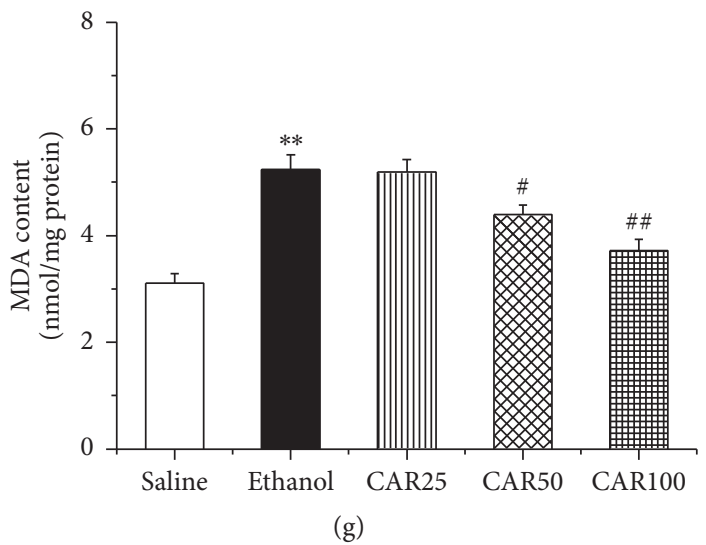

FIGURE 7: Effects of carvacrol on oxidative stress biomarkers of the hippocampus in ethanol-treated C57BL/6 mice. (a)-(g) showed the effects of carvacrol on activities of CuZn-SOD, Mn-SOD, SOD, GSH, GSH-PX, CAT, and MDA level from different group, respectively. Saline: saline group; Ethanol: ethanol group; CAR25: ethanol and carvacrol $25 \mathrm{mg} / \mathrm{kg}$-treated group; CAR50: ethanol and carvacrol $50 \mathrm{mg} / \mathrm{kg}$-treated group; CAR100: ethanol and carvacrol $100 \mathrm{mg} / \mathrm{kg}$-treated group. The data are expressed as the mean \pm SEM $(n=6$ per group $) .{ }^{* *} P<0.01$ compared to saline group; ${ }^{\#} P<0.05$ and ${ }^{\# \#} P<0.01$ compared to ethanol group. 
Tissue

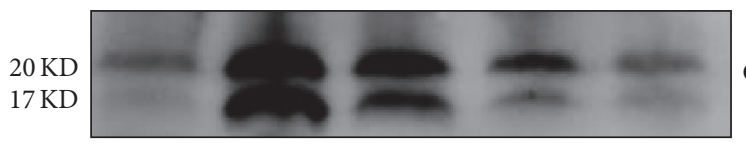

Caspase-3

$43 \mathrm{KD}$

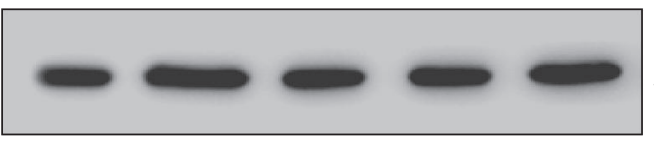
$\beta$-Actin
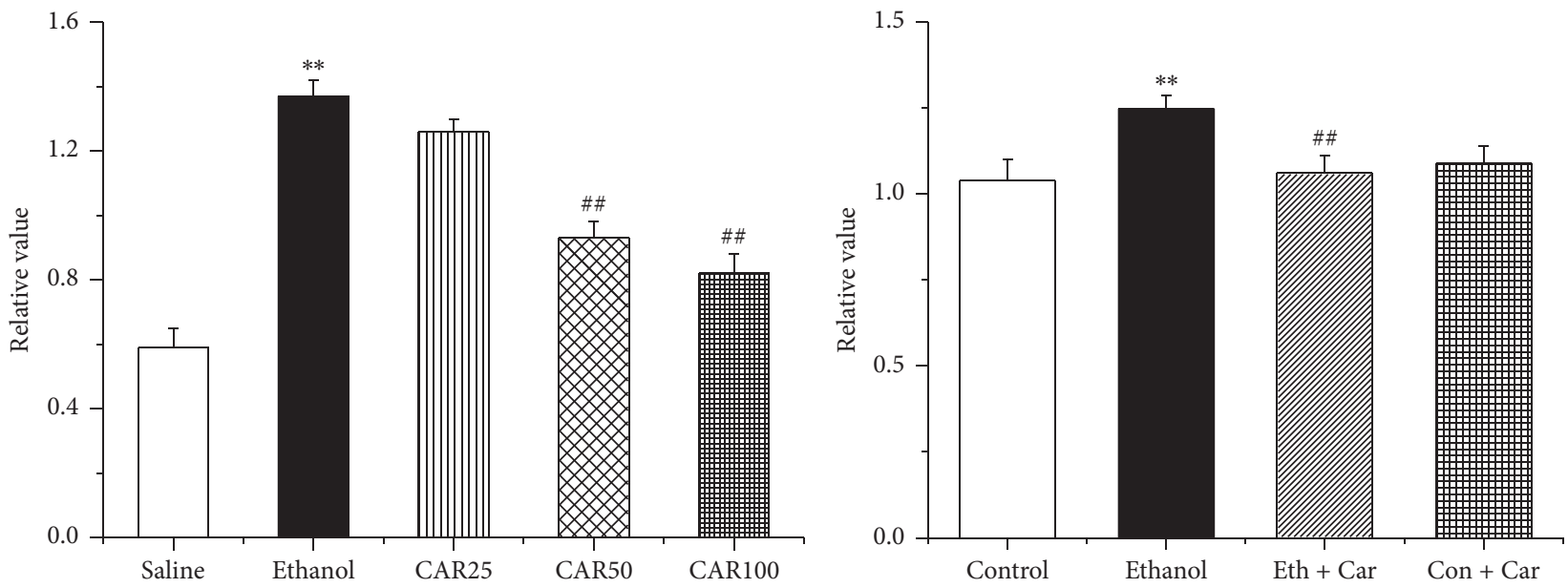

(a)

Tissue

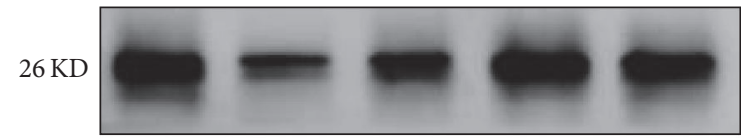
Bcl-2

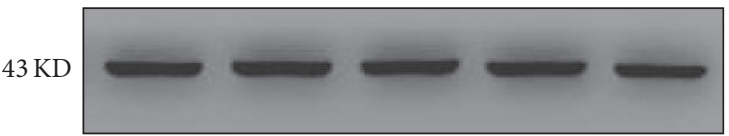

$\beta$-Actin

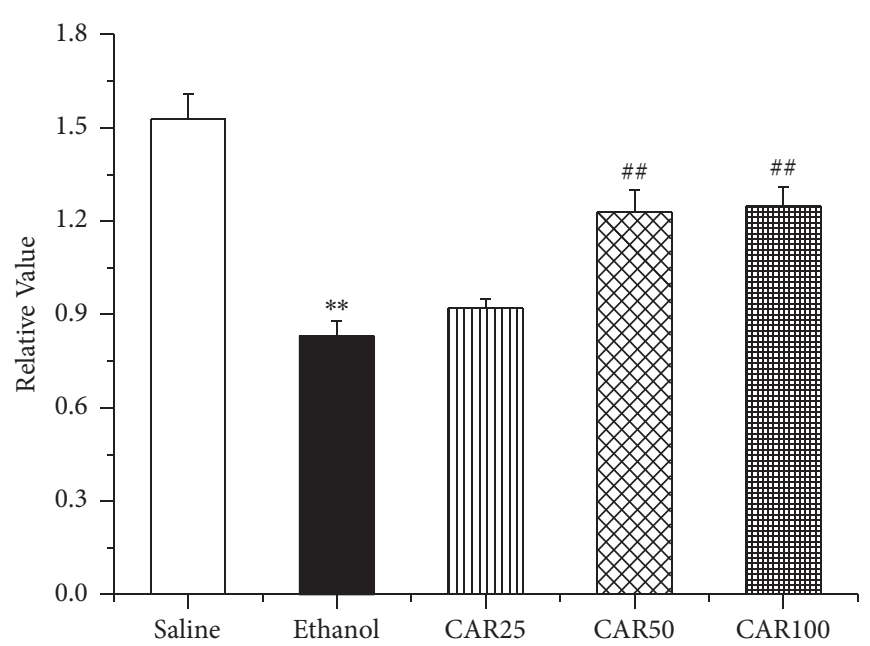

Cell

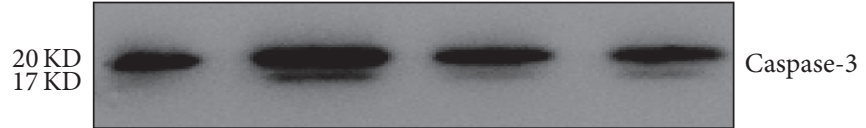

$43 \mathrm{KD}$

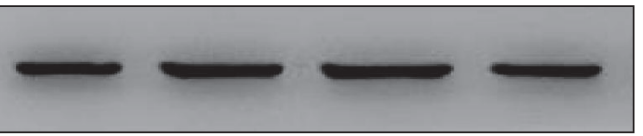

$\beta$-Actin
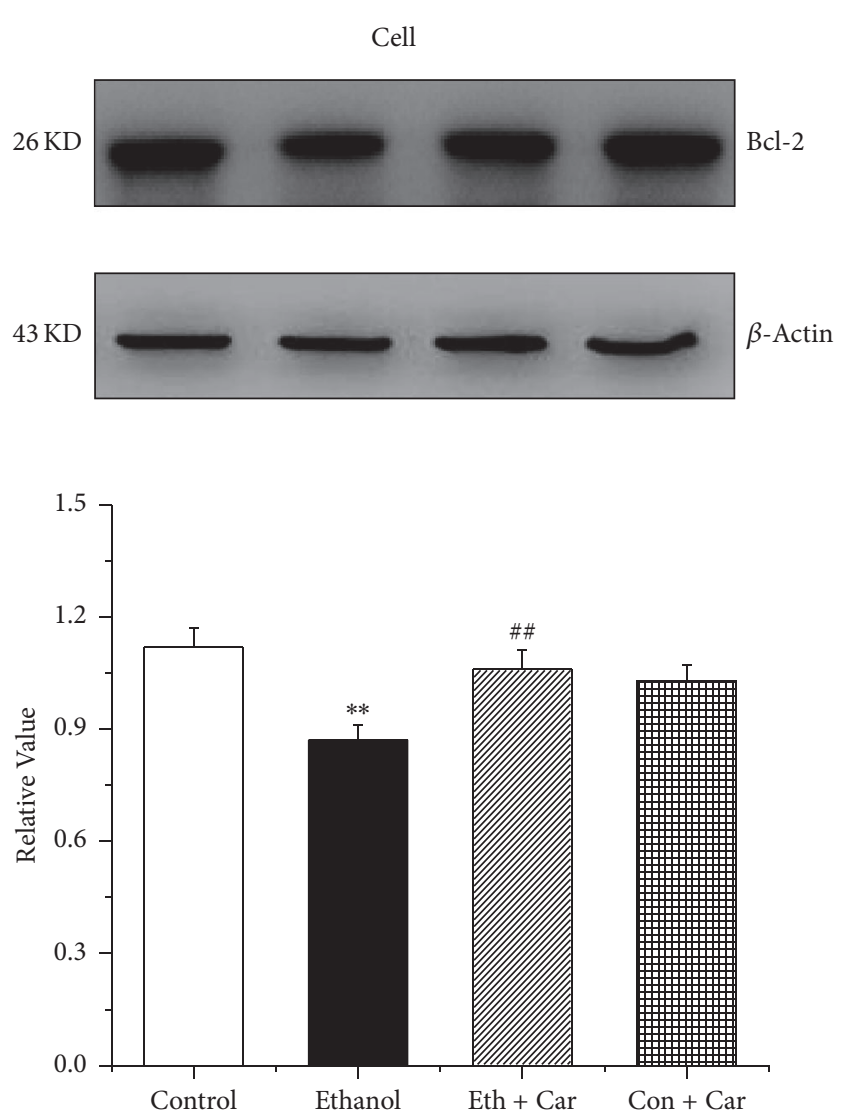

(b)

FIgURE 8: Continued. 
Tissue
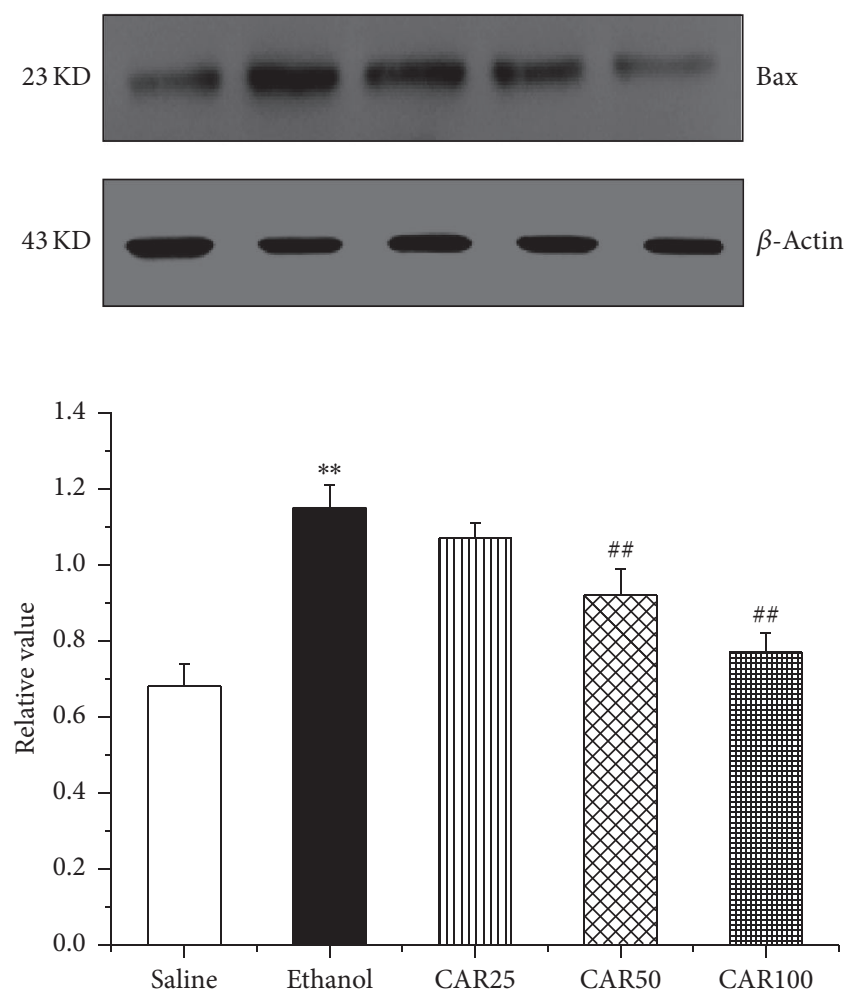

Cell
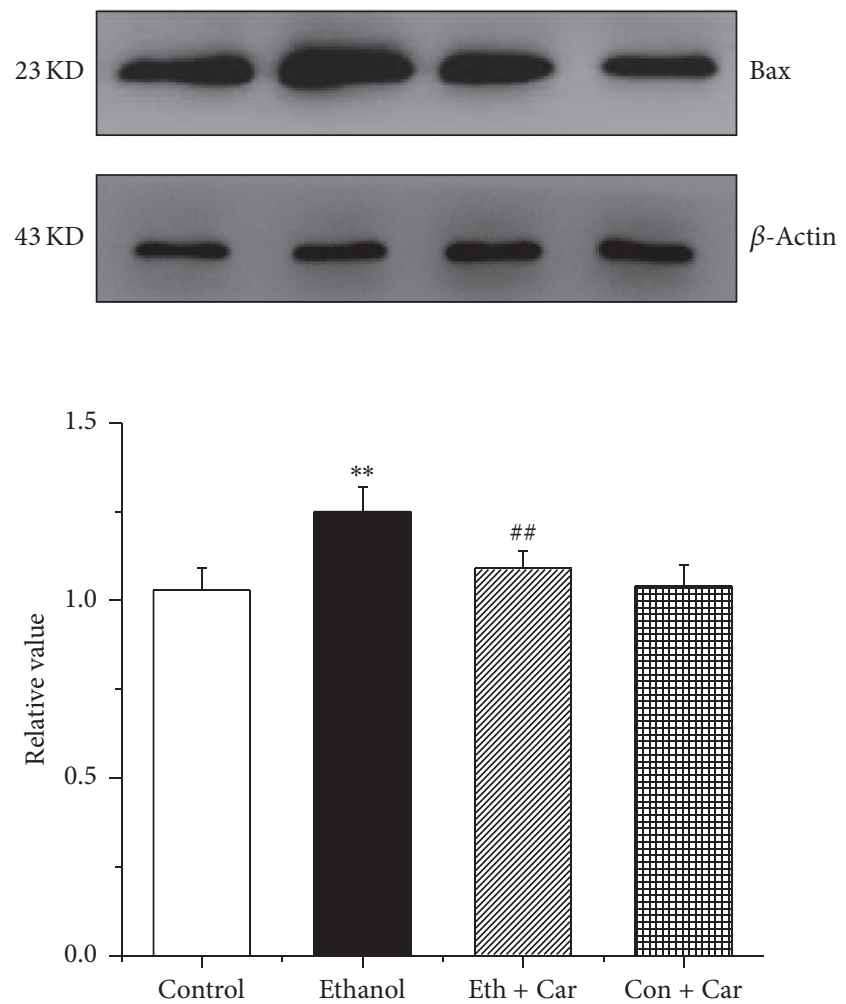

(c)

FIGURE 8: Carvacrol altered the protein expression of caspase-3, Bcl-2, and Bax of the hippocampus in ethanol-treated C57BL/6 mice and hippocampal neurons. (a), (b), and (c) show the quantitative analysis of the protein levels of caspase-3, Bcl-2, and Bax in mouse hippocampi and hippocampal neurons, respectively. The data were normalized to the loading control GAPDH. The data are expressed as the mean \pm SEM ( $n=6$ per group). ${ }^{* *} P<0.01$ compared to saline group; ${ }^{\# \#} P<0.01$ compared to ethanol group.

alleviates hippocampal neuronal impairment by antioxidative and antiapoptotic effects, which is likely related to the mitochondrial and mitogen-activated protein kinase signal pathway.

The ethological [32], morphological [33], chemical, and molecular biological determinations showed that exposure to ethanol for 4 weeks can induce oxidative stress [34] and result in mice hippocampal neuronal apoptosis [35], which finally leads to cognitive dysfunction [36]. Our study showed that carvacrol treatment partially reversed learning and memory deficits in the ethanol-induced model by decreasing escape latency and increasing number of times of platform passing. At the same time, Nissl staining and NeuN immunohistochemistry showed that carvacrol treatment at doses of 50 and $100 \mathrm{mg} / \mathrm{kg}$ significantly reduced the neuronal apoptosis in vivo. Furthermore, $0.8 \mathrm{mM}$ of carvacrol significantly improves cell viability of ethanol-induced hippocampal neuron in vitro. These findings indicate that carvacrol alleviates learning and memory deficits via inhibiting hippocampal neuronal apoptosis.

The compelling evidence supported that oxidative damage, an important index for evaluating the neuronal injury caused by ethanol, plays a crucial role in neuropathologic lesions [37-39]. Therefore, we speculate that carvacrol partially reversed neuronal injury in the ethanol-induced model by decreasing hippocampal neuronal oxidative stress. Our results illustrated that the CuZn-SOD, Mn-SOD, SOD, GSH, GSH-PX, and CAT activities decreased and the level of MDA increased in ethanol-treated mice, and carvacrol partially reversed the changes. Therefore, the reduction of oxidative stress and enhancement of antioxidative stress might have an important role in the protective effects of carvacrol on ethanol-induced neuron injury.

It is well known that oxidative stress can induce apoptosis [40]. However, apoptosis pathways include the mitochondrial pathway, the death receptor pathway, and the endoplasmic reticulum stress pathway. We next sought to examine the signaling pathways that participated in the induction of hippocampal neuronal apoptosis induced by ethanol. The mitochondrial pathway of apoptosis began with the permeabilisation of the mitochondrial outer membrane. Furthermore, mitochondrial membrane permeabilisation is regulated by Bcl-2 family members, which can be subdivided into antiapoptotic members such as Bcl-2 and proapoptotic species 
Tissue
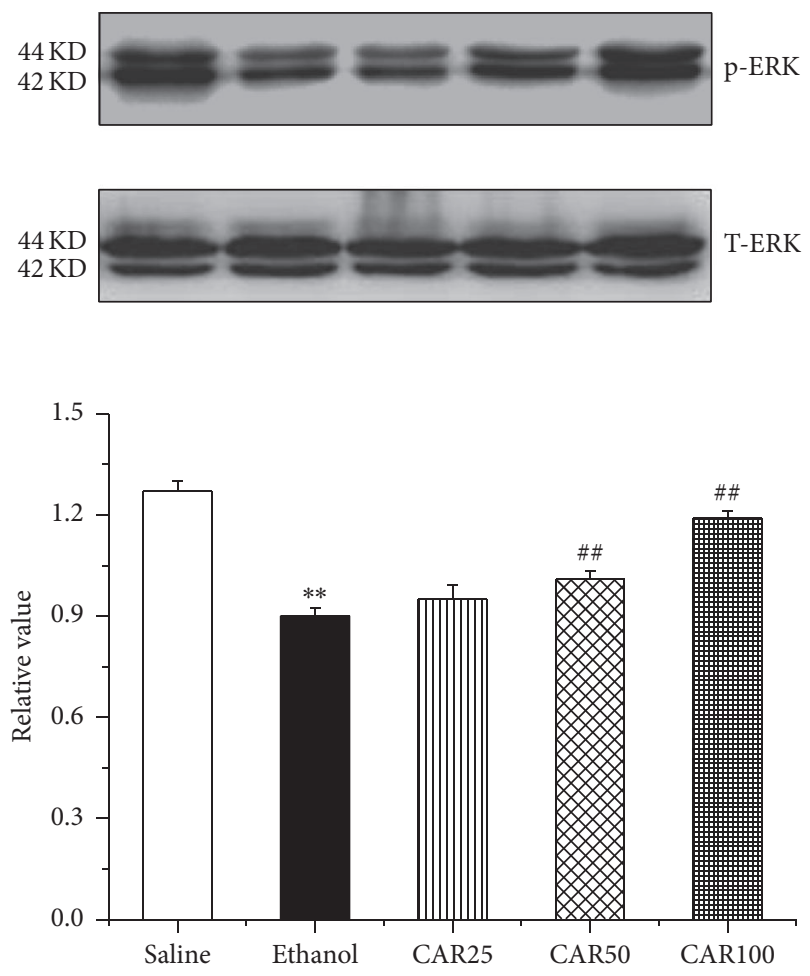

Tissue

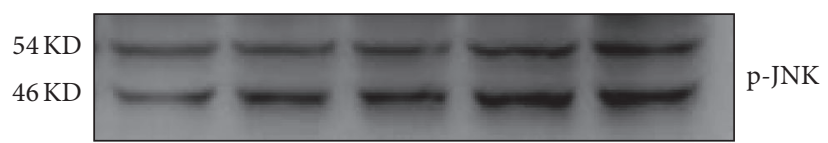

54 KD

$46 \mathrm{KD}$

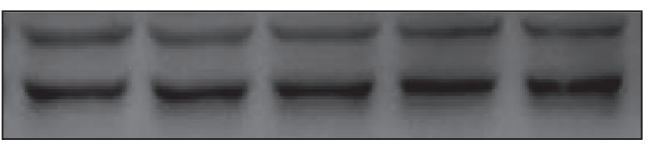

T-JNK

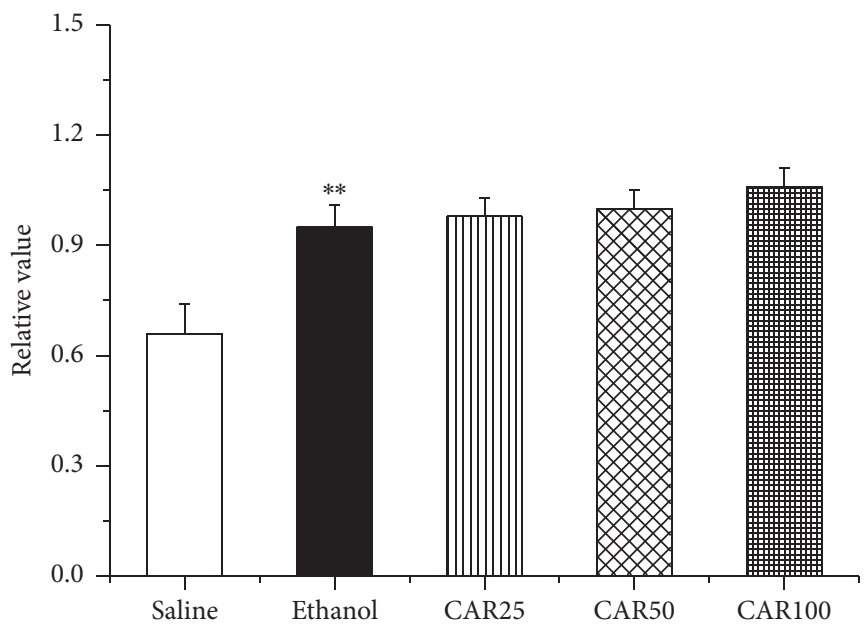

(b)

(a)
Cell

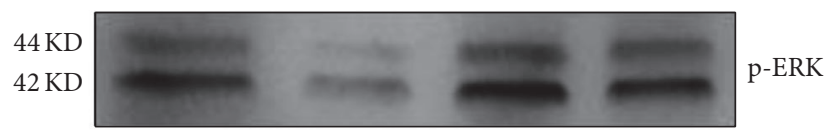

$44 \mathrm{KD}$ $42 \mathrm{KD}$

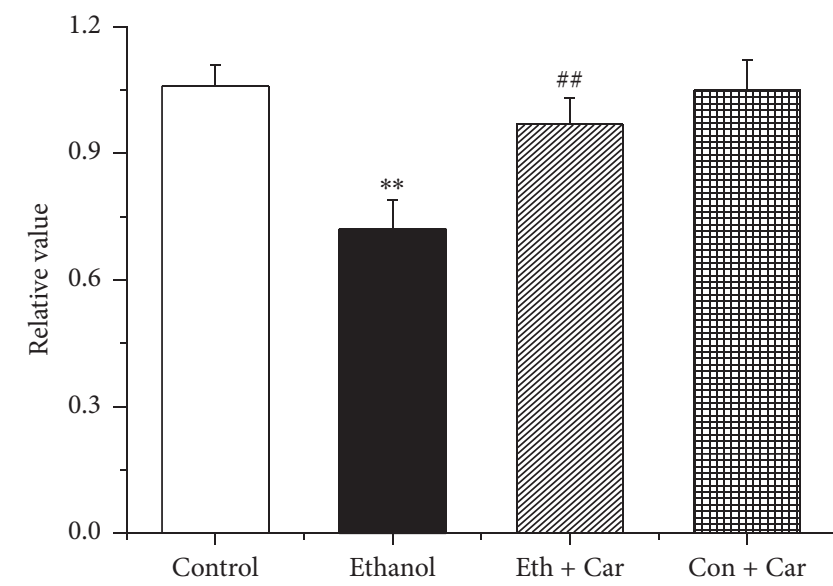

Cell
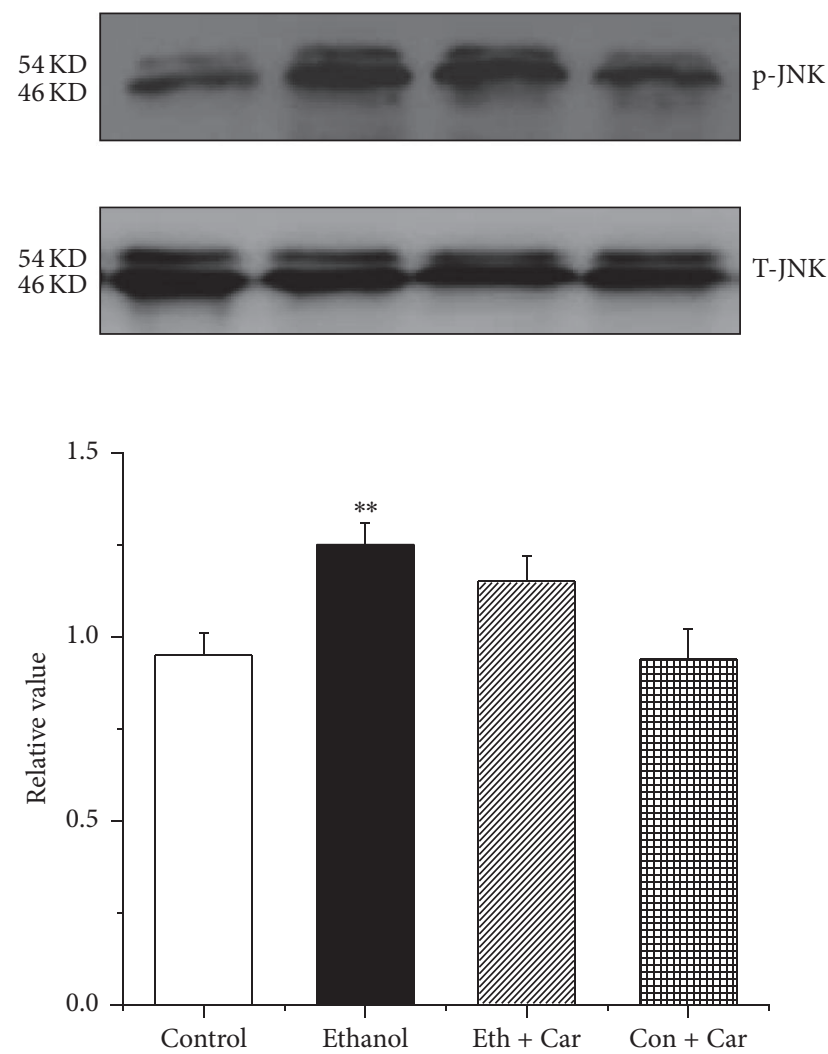

FIgure 9: Continued. 

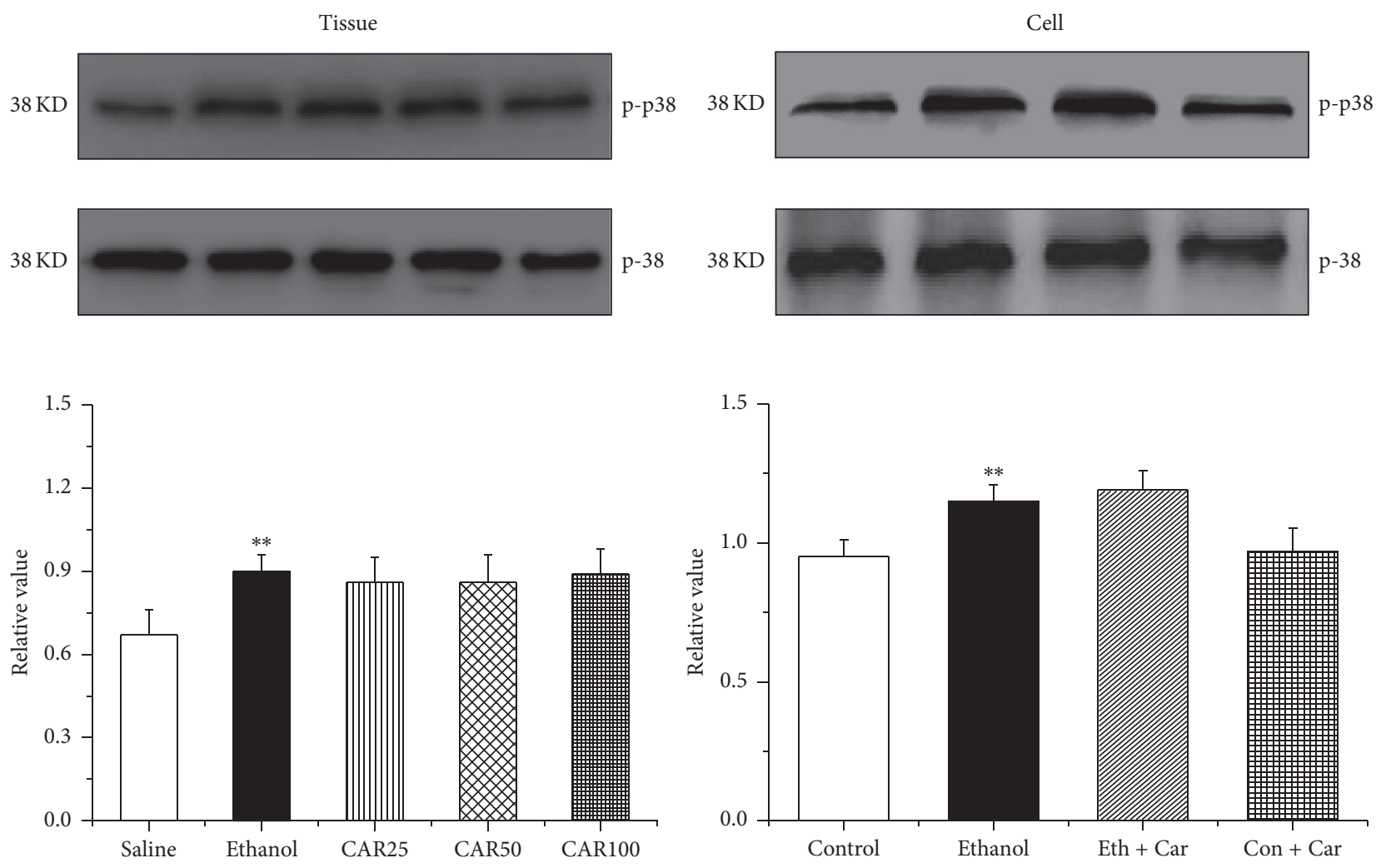

(c)

Figure 9: Carvacrol influenced the protein levels of MAPK cascades of the hippocampus of ethanol-treated C57BL/6 mice and ethanoltreated hippocampal neurons. (a), (b), and (c) are the quantitative analyses of the protein levels of p-ERK-1/2, p-JNK, and p-p38, which were normalized to ERK-1/2, JNK, and p38, respectively. The data are expressed as the mean \pm SEM ( $n=6$ per group). ${ }^{* *} P<0.01$ compared to saline group; ${ }^{\# \#} P<0.01$ compared to ethanol group.

such as Bax [41]. In our study, high concentration of ethanol could upregulate the expression of Bax and downregulate the expression of Bcl-2. Carvacrol could further weaken the effect of ethanol. Thus, it was speculated that carvacrol protects hippocampal neuron from apoptosis through mitochondrial pathway associated with the upregulation of antiapoptotic protein Bcl-2 and downregulation of proapoptotic protein Bax.

Alteration of the MAPKs signal pathway has been observed in ethanol-induced cell death $[42,43]$. We found that ethanol inhibited the phosphorylation of ERK-1/2, which is in agreement with previous research in which ethanol exposure decreased ERK phosphorylation [44] but increased that of p-JNK and p-p38 in ethanol-treated mouse hippocampus [22]. However, carvacrol reversed the phosphorylation of ERK-1/2 change induced by ethanol and had no influence on p-JNK and p-p38 expression. The results indicate that activation of ERK-1/2 signaling pathway is a possible neuroprotective mechanism of carvacrol following ethanol exposure.

Our data in the present study indicate that the protective effects of carvacrol on ethanol-induced cognitive dysfunction of the mice and hippocampal neuronal impairment might be associated with antioxidative and antiapoptotic effects as well as the modulation of MAPK cascades and mitochondrial pathway. Since carvacrol is a natural product with a proven record of safe human administration, it is possible that carvacrol may be regarded as a promising drug in clinical therapy.

\section{Competing Interests}

The authors declare that there are no competing interests regarding the publication of this paper.

\section{Authors' Contributions}

Peng Wang, Lihui Qu, Hui Ding, Qianlong Zhang, and Yonggang Cao participated in the experimental studies. Qian Luo, Juan Yu, Ruxia Liu, and Hui Qiao analyzed the data, and Hui Zhu wrote the paper.

\section{Acknowledgments}

This study was supported by Natural Science Foundation of Heilongiiang Province of China (QC2012C032) and Seed Fund of Harbin Medical University (Daqing) (DQ2014-04). 

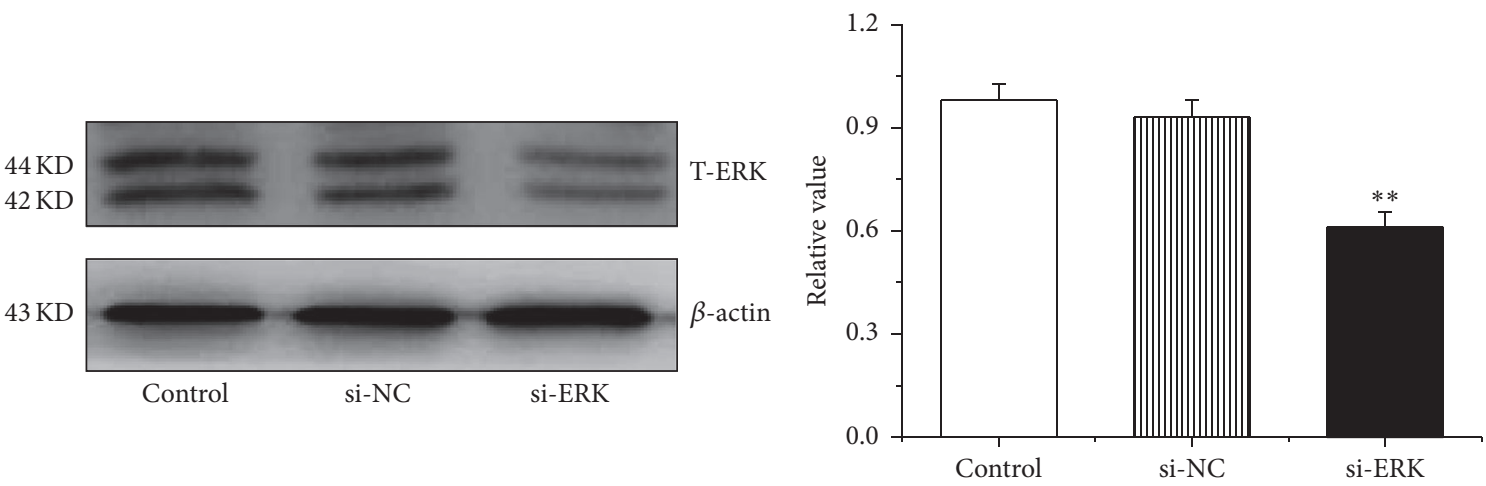

(a)
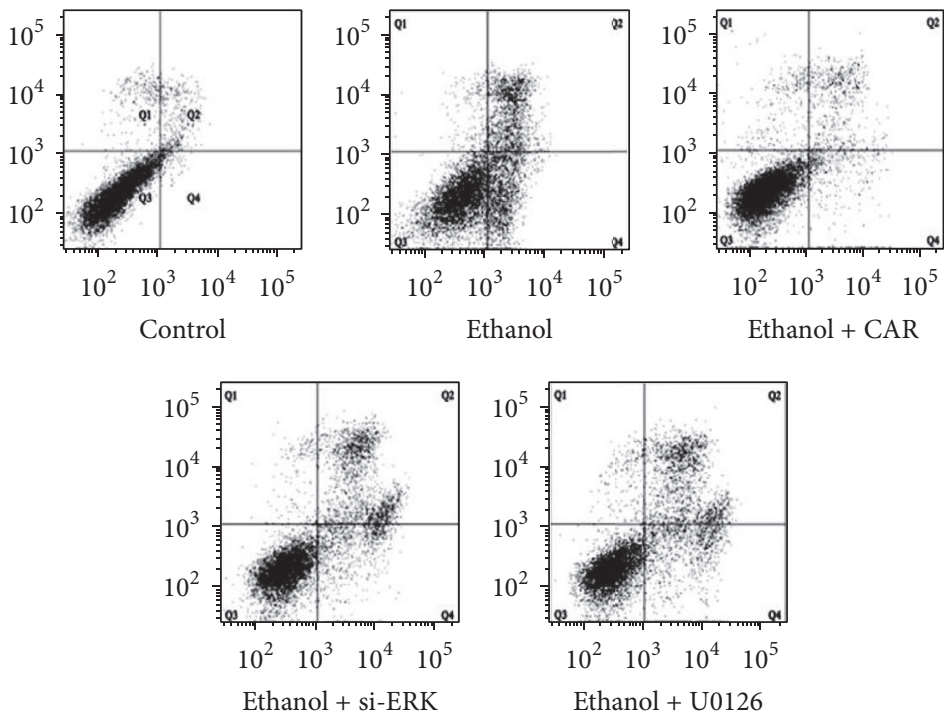

(b)

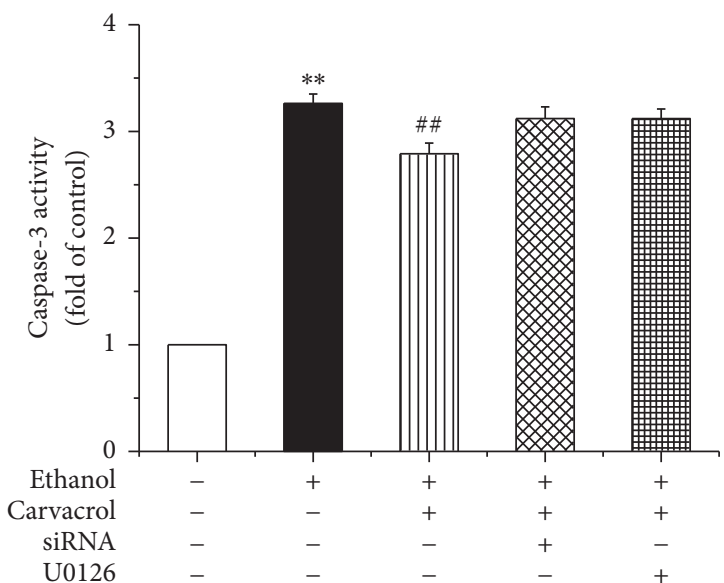

(c)

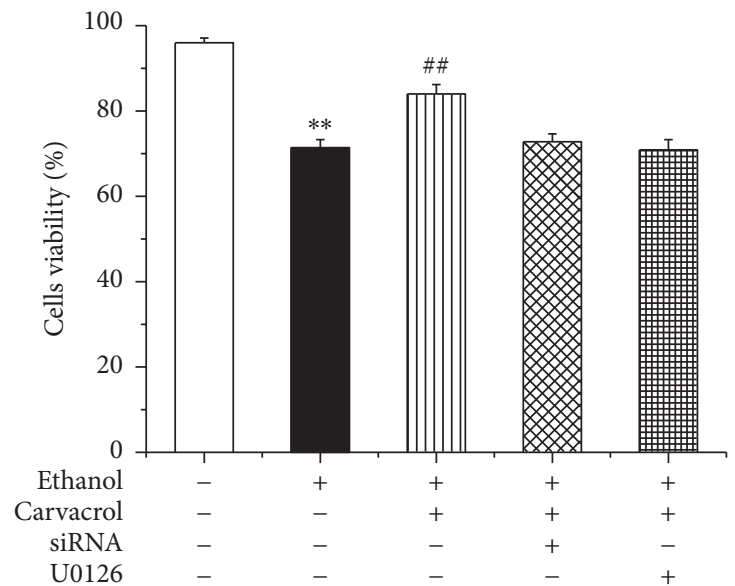

(d)

FIGURE 10: Silence ERK pathway partially repeal the antiapoptosis effects of carvacrol. (a) The efficiency and specificity of siRNA directed against ERK. (b)-(d) Ethanol increases hippocampal neurons apoptotic rate and caspase-3 activity as well as decreasing cell viability compared with control group. These proapoptotic effects can be reversed by carvacrol. However, when it is silence or block ERK pathway, carvacrol antiapoptotic effects can be partially repealed. The data are expressed as the mean $\pm \operatorname{SEM}\left(n=6\right.$ per group). ${ }^{* *} P<0.01$ compared to control group; ${ }^{\# \#} P<0.01$ compared to ethanol group. 


\section{References}

[1] A. M. White, D. B. Matthews, and P. J. Best, "Ethanol, memory, and hippocampal function: a review of recent findings," Hippocampus, vol. 10, no. 1, pp. 88-93, 2000.

[2] M. Roberto, T. E. Nelson, C. L. Ur, and D. L. Gruol, "Long-term potentiation in the rat hippocampus is reversibly depressed by chronic intermittent ethanol exposure," Journal of Neurophysiology, vol. 87, no. 5, pp. 2385-2397, 2002.

[3] M. Tateno and T. Saito, "Biological studies on alcohol-induced neuronal damage," Psychiatry Investigation, vol. 5, no. 1, pp. 21$27,2008$.

[4] R. Guo and R. Jun, "Alcohol and acetaldehyde in public health: from marvel to menace," International Journal of Environmental Research and Public Health, vol. 7, no. 4, pp. 1285-1301, 2010.

[5] F. Fadda and Z. L. Rossetti, "Chronic ethanol consumption: from neuroadaptation to neurodegeneration," Progress in Neurobiology, vol. 56, no. 4, pp. 385-431, 1998.

[6] D. F. Wozniak, R. E. Hartman, M. P. Boyle et al., "Apoptotic neurodegeneration induced by ethanol in neonatal mice is associated with profound learning/memory deficits in juveniles followed by progressive functional recovery in adults," Neurobiology of Disease, vol. 17, no. 3, pp. 403-414, 2004.

[7] J. D. Cushman, M. D. Moore, N. S. Jacobs, R. W. Olsen, and M. S. Fanselow, "Behavioral pharmacogenetic analysis on the role of the $\alpha 4 \mathrm{GABA}_{\mathrm{A}}$ receptor subunit in the ethanol-mediated impairment of hippocampus-dependent contextual learning," Alcoholism: Clinical and Experimental Research, vol. 35, no. 11, pp. 1948-1959, 2011.

[8] C. Harper, "The neuropathology of alcohol-specific brain damage, or does alcohol damage the brain?" Journal of Neuropathology and Experimental Neurology, vol. 57, no. 2, pp. 101-110, 1998.

[9] T. E. Nelson, C. L. Ur, and D. L. Gruol, "Chronic intermittent ethanol exposure alters CA1 synaptic transmission in rat hippocampal slices," Neuroscience, vol. 94, no. 2, pp. 431-442, 1999.

[10] M. E. Bates, S. C. Bowden, and D. Barry, "Neurocognitive impairment associated with alcohol use disorders: implications for treatment," Experimental and Clinical Psychopharmacology, vol. 10, no. 3, pp. 193-212, 2002.

[11] R. P. Vetreno, J. M. Hall, and L. M. Savage, "Alcohol-related amnesia and dementia: animal models have revealed the contributions of different etiological factors on neuropathology, neurochemical dysfunction and cognitive impairment," Neurobiology of Learning and Memory, vol. 96, no. 4, pp. 596-608, 2011.

[12] Z. Jin, A. K. Bhandage, I. Bazov et al., "Selective increases of AMPA, NMDA, and kainate receptor subunit mRNAs in the hippocampus and orbitofrontal cortex but not in prefrontal cortex of human alcoholics," Frontiers in Cellular Neuroscience, vol. 8, article no. 11, 2014.

[13] A. Y. Sun, M. Ingelman-Sundberg, E. Neve et al., "Ethanol and oxidative stress," Alcoholism: Clinical and Experimental Research, vol. 25, no. 5, pp. 237S-243S, 2001.

[14] U. O. Turkcu, A. Bilgihan, G. Biberoglu, and O. M. Caglar, "Carnosine supplementation protects rat brain tissue against ethanol-induced oxidative stress," Molecular and Cellular Biochemistry, vol. 339, no. 1-2, pp. 55-61, 2010.

[15] S. A. Shah, G. H. Yoon, and M. O. Kim, "Protection of the developing brain with anthocyanins against ethanol-induced oxidative stress and neurodegeneration," Molecular Neurobiology, vol. 51, no. 3, pp. 1278-1291, 2015.
[16] A. M. Antonio and M. J. Druse, "Antioxidants prevent ethanolassociated apoptosis in fetal rhombencephalic neurons," Brain Research, vol. 1204, pp. 16-23, 2008.

[17] Z. Wang, T. Yao, and Z. Song, "Chronic alcohol consumption disrupted cholesterol homeostasis in rats: down-regulation of low-density lipoprotein receptor and enhancement of cholesterol biosynthesis pathway in the liver," Alcoholism: Clinical and Experimental Research, vol. 34, no. 3, pp. 471-478, 2010.

[18] V. Tiwari and K. Chopra, "Resveratrol abrogates alcoholinduced cognitive deficits by attenuating oxidative-nitrosative stress and inflammatory cascade in the adult rat brain," Neurochemistry International, vol. 62, no. 6, pp. 861-869, 2013.

[19] B. Scolaro, D. Delwing-De Lima, J. G. P. Da Cruz, and D. Delwing-Dal Magro, "Mate tea prevents oxidative stress in the blood and hippocampus of rats with acute or chronic ethanol administration," Oxidative Medicine and Cellular Longevity, vol. 2012, Article ID 314758, 8 pages, 2012.

[20] M. B. Heaton, M. Paiva, and K. Siler-Marsiglio, "Ethanol influences on bax translocation, mitochondrial membrane potential, and reactive oxygen species generation are modulated by vitamin E and brain-derived neurotrophic factor," Alcoholism: Clinical and Experimental Research, vol. 35, no. 6, pp. 1122-1133, 2011.

[21] Y. Zhong, G. Dong, H. Luo et al., "Induction of brain CYP2E1 by chronic ethanol treatment and related oxidative stress in hippocampus, cerebellum, and brainstem," Toxicology, vol. 302, no. 2-3, pp. 275-284, 2012.

[22] B. Zhao, Y. Wang, Y. Li et al., "Differential phosphorylation of NMDAR1-CaMKII-MAPKs in the rat nucleus accumbens following chronic ethanol exposure," Neuroscience Letters, vol. 597, pp. 60-65, 2015.

[23] S.-Z. Cui, S.-J. Wang, J. Li et al., "Alteration of synaptic plasticity in rat dorsal striatum induced by chronic ethanol intake and withdrawal via ERK pathway," Acta Pharmacologica Sinica, vol. 32, no. 2, pp. 175-181, 2011.

[24] N. Kirimer, K. H. C. Başer, and G. Tümen, "Carvacrol-rich plants in Turkey," Chemistry of Natural Compounds, vol. 31, no. 1, pp. 37-41, 1995.

[25] B. Aristatile, K. S. Al-Numair, C. Veeramani, and K. V. Pugalendi, "Effect of carvacrol on hepatic marker enzymes and antioxidant status in d-galactosamine-induced hepatotoxicity in rats," Fundamental and Clinical Pharmacology, vol. 23, no. 6, pp. 757-765, 2009.

[26] M. Sökmen, J. Serkedjieva, D. Daferera et al., "In vitro antioxidant, antimicrobial, and antiviral activities of the essential oil and various extracts from herbal parts and callus cultures of Origanum acutidens," Journal of Agricultural and Food Chemistry, vol. 52, no. 11, pp. 3309-3312, 2004.

[27] P. Landa, L. Kokoska, M. Pribylova, T. Vanek, and P. Marsik, "In vitro anti-inflammatory activity of carvacrol: inhibitory effect on COX-2 catalyzed prostaglandin $\mathrm{E}_{2}$ biosynthesis," Archives of Pharmacal Research, vol. 32, no. 1, pp. 75-78, 2009.

[28] K. H. C. Baser, "Biological and pharmacological activities of carvacrol and carvacrol bearing essential oils," Current Pharmaceutical Design, vol. 14, no. 29, pp. 3106-3119, 2008.

[29] M. Jukic, O. Politeo, M. Maksimovic, M. Milos, and M. Milos, "In vitro acetylcholinesterase inhibitory properties of thymol, carvacrol and their derivatives thymoquinone and thymohydroquinone," Phytotherapy Research, vol. 21, no. 3, pp. 259-261, 2007.

[30] H. Yu, Z.-L. Zhang, J. Chen et al., "Carvacrol, a foodadditive, provides neuroprotection on focal cerebral ischemia/ 
reperfusion injury in mice," PLoS ONE, vol. 7, no. 3, Article ID e33584, 2012.

[31] X. Wang, Z. Ke, G. Chen et al., "Cdc42-dependent activation of NADPH oxidase is involved in Ethanol-Induced neuronal oxidative stress," PLoS ONE, vol. 7, no. 5, Article ID e38075, 2012.

[32] T. Hashemi Nosrat Abadi, L. Vaghef, S. Babri, M. MahmoodAlilo, and M. Beirami, "Effects of different exercise protocols on ethanol-induced spatial memory impairment in adult male rats," Alcohol, vol. 47, no. 4, pp. 309-316, 2013.

[33] L. M. Savage, P. M. Candon, and H. L. Hohmann, "Alcoholinduced brain pathology and behavioral dysfunction: using animal model to examine sex differences," Alcoholism: Clinical and Experimental Research, vol. 24, no. 4, pp. 465-475, 2000.

[34] S. K. Das, K. R. Hiran, S. Mukherjee, and D. M. Vasudevan, "Oxidative stress is the primary event: effects of ethanol consumption in brain," Indian Journal of Clinical Biochemistry, vol. 22, no. 1, pp. 99-104, 2007.

[35] B. M. Ku, Y. K. Lee, J. Y. Jeong et al., "Ethanol-induced oxidative stress is mediated by p38 MAPK pathway in mouse hippocampal cells," Neuroscience Letters, vol. 419, no. 1, pp. 6467, 2007.

[36] D. B. Matthews and A. L. Morrow, "Effects of acute and chronic ethanol exposure on spatial cognitive processing and hippocampal function in the rat," Hippocampus, vol. 10, no. 1 , pp. 122-130, 2000.

[37] J. Haorah, S. H. Ramirez, N. Floreani, S. Gorantla, B. Morsey, and Y. Persidsky, "Mechanism of alcohol-induced oxidative stress and neuronal injury," Free Radical Biology and Medicine, vol. 45, no. 11, pp. 1542-1550, 2008.

[38] G. Chen, C. Ma, K. A. Bower, X. Shi, Z. Ke, and J. Luo, "Ethanol promotes endoplasmic reticulum stress-induced neuronal death: involvement of oxidative stress," Journal of Neuroscience Research, vol. 86, no. 4, pp. 937-946, 2008.

[39] N. Phunchago, J. Wattanathorn, and K. Chaisiwamongkol, "Tiliacora triandra, an anti-intoxication plant, improves memory impairment, neurodegeneration, cholinergic function, and oxidative stress in hippocampus of ethanol dependence rats," Oxidative Medicine and Cellular Longevity, vol. 2015, Article ID 918426, 9 pages, 2015.

[40] K. Kannan and S. K. Jain, "Oxidative stress and apoptosis," Pathophysiology, vol. 7, no. 3, pp. 153-163, 2000.

[41] M. D. Esposti and C. Dive, "Mitochondrial membrane permeabilisation by Bax/Bak," Biochemical and Biophysical Research Communications, vol. 304, no. 3, pp. 455-461, 2003.

[42] H. S. G. Kalluri and M. K. Ticku, "Ethanol-mediated inhibition of mitogen-activated protein kinase phosphorylation in mouse brain," European Journal of Pharmacology, vol. 439, no. 1-3, pp. 53-58, 2002.

[43] P. P. Sanna, C. Simpson, R. Lutjens, and G. Koob, "ERK regulation in chronic ethanol exposure and withdrawal," Brain Research, vol. 948, no. 1-2, pp. 186-191, 2002.

[44] B. M. Ku, Y. K. Lee, J. Y. Jeong et al., "Ethanol-induced oxidative stress is mediated by $\mathrm{p} 38$ MAPK pathway in mouse hippocampal cells," Neuroscience Letters, vol. 419, no. 1, pp. 6467, 2007. 


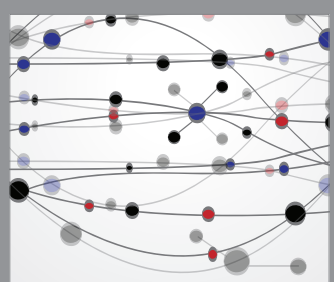

The Scientific World Journal
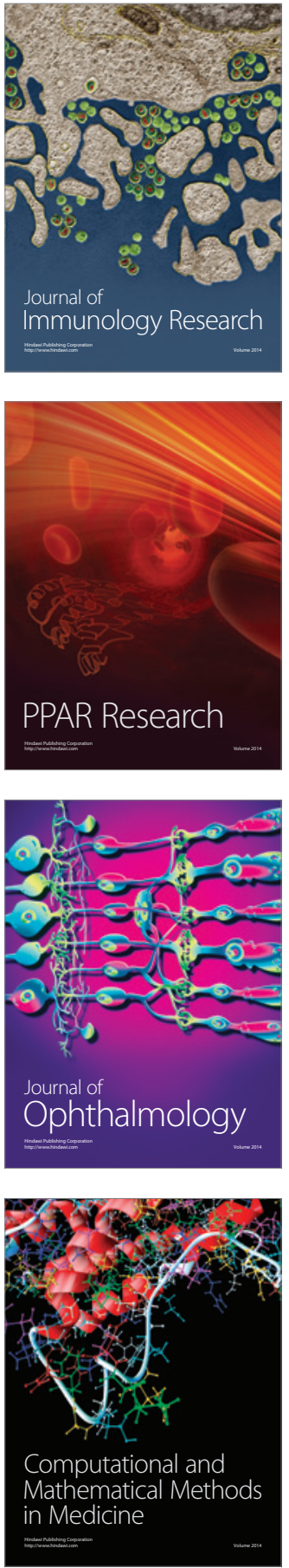

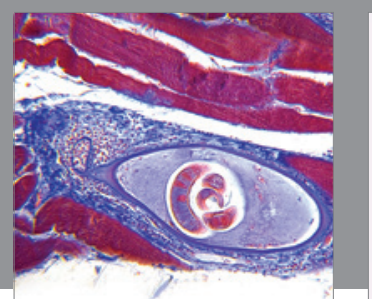

Gastroenterology Research and Practice
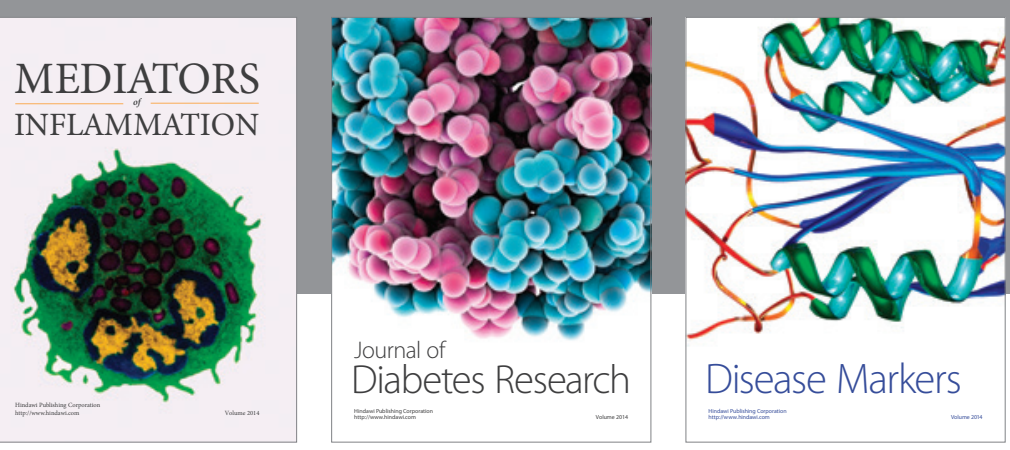

Disease Markers

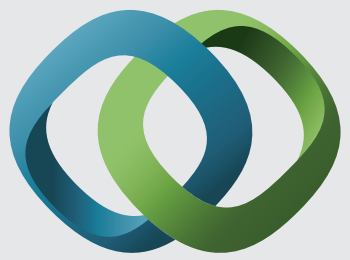

\section{Hindawi}

Submit your manuscripts at

https://www.hindawi.com
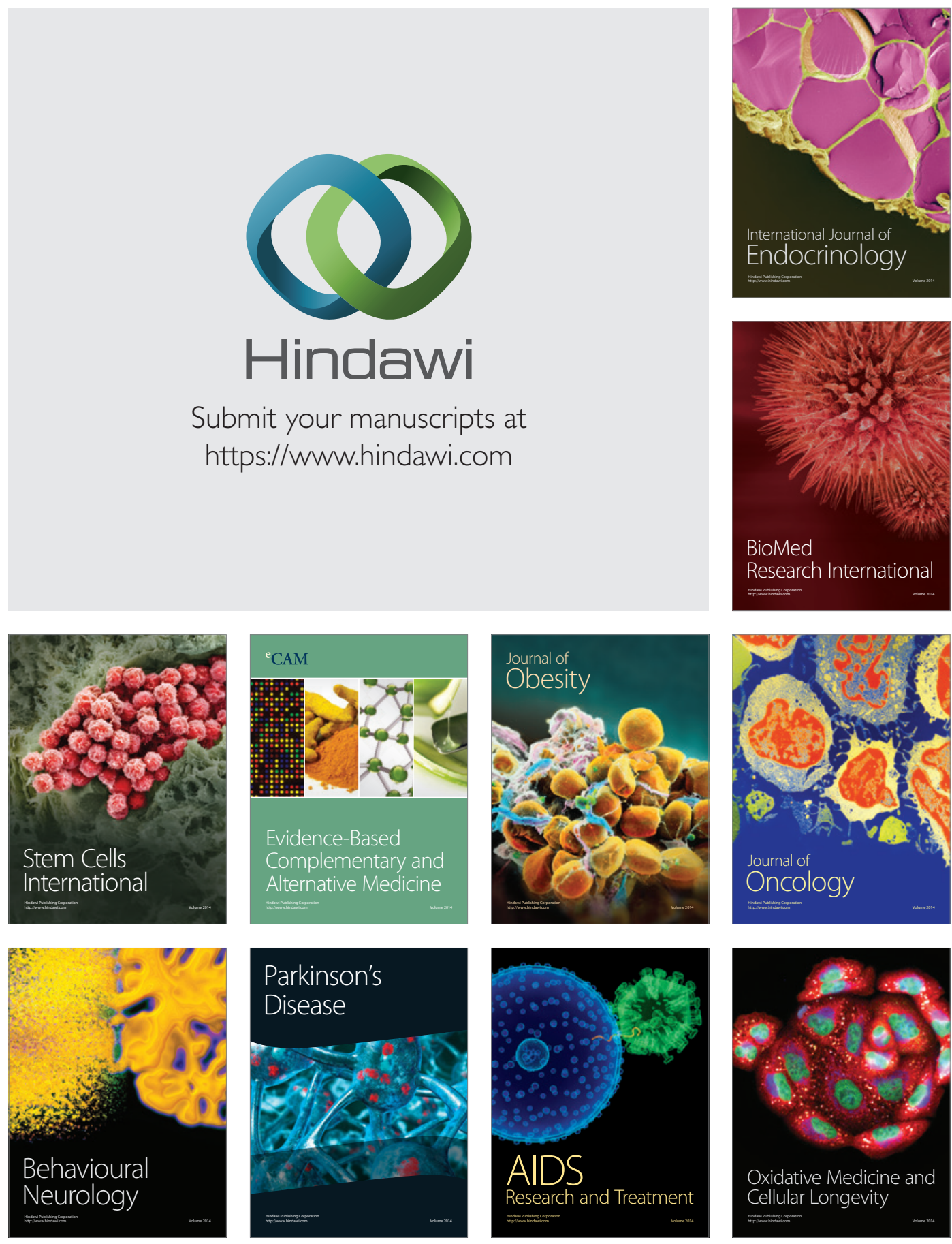